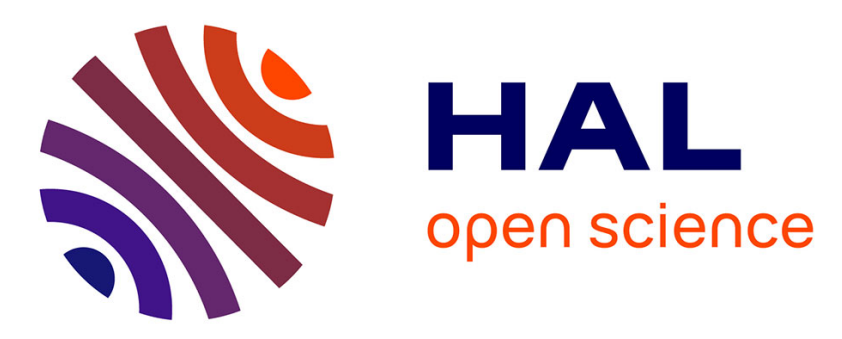

\title{
Tryptophan-mediated Dimerization of the TssL Transmembrane Anchor Is Required for Type VI Secretion System Activity
}

Abdelrahim Zoued, Jean-Pierre Duneau, Eric Durand, Alexandre España, Laure Journet, Françoise Guerlesquin, E. Cascales

\section{To cite this version:}

Abdelrahim Zoued, Jean-Pierre Duneau, Eric Durand, Alexandre España, Laure Journet, et al.. Tryptophan-mediated Dimerization of the TssL Transmembrane Anchor Is Required for Type VI Secretion System Activity. Journal of Molecular Biology, 2018, 430 (7), pp.987 - 1003. 10.1016/j.jmb.2018.02.008 . hal-01780763

\section{HAL Id: hal-01780763 \\ https://hal-amu.archives-ouvertes.fr/hal-01780763}

Submitted on 27 Apr 2018

HAL is a multi-disciplinary open access archive for the deposit and dissemination of scientific research documents, whether they are published or not. The documents may come from teaching and research institutions in France or abroad, or from public or private research centers.
L'archive ouverte pluridisciplinaire HAL, est destinée au dépôt et à la diffusion de documents scientifiques de niveau recherche, publiés ou non, émanant des établissements d'enseignement et de recherche français ou étrangers, des laboratoires publics ou privés. 


\section{Tryptophan-mediated dimerization of the TssL transmembrane anchor is required for Type VI secretion system activity}

Abdelrahim Zoued ${ }^{\dagger}$, Jean-Pierre Duneau*, Eric Durand, Alexandre P. España ${ }^{\S}$, Laure Journet, Françoise Guerlesquin*, and Eric Cascales*

Running title: Dimerization of the TssL transmembrane helix

Laboratoire d'Ingénierie des Systèmes Macromoléculaires (LISM), Institut de Microbiologie de la Méditerranée (IMM), Aix-Marseille Université, CNRS, UMR 7255, 31 chemin Joseph Aiguier, 13402 Marseille Cedex 20, France

${ }^{\dagger}$ Current address: Howard Hughes Medical Institute, Brigham and Women's Hospital, Division of Infectious Diseases and Harvard Medical School, Department of Microbiology and Immunobiology, Boston, Massachusetts, USA.

$\S$ Current address: Technological Advances for Genomics and Clinics laboratory (TAGC, U1090), Aix-Marseille Univ. - Institut National de la Santé et de la Recherche Médicale (INSERM), 163 Avenue de Luminy, 13288 Marseille Cedex 09, France.

* To whom correspondence should be addressed:

Jean-Pierre Duneau (duneau@imm.cnrs.fr)

Françoise Guerlesquin (guerlesq@imm.cnrs.fr)

Eric Cascales (cascales@imm.cnrs.fr) 


\section{Abstract}

3 The Type VI secretion system (T6SS) is a multiprotein complex used by bacteria to

4 deliver effectors into target cells. The T6SS comprises a bacteriophage-like contractile

5 tail structure anchored to the cell envelope by a membrane complex constituted of the

6 TssJ outer membrane lipoprotein and the TssL and TssM inner membrane proteins.

7 TssJ establishes contact with the periplasmic domain of TssM whereas the 8 transmembrane segments of TssM and its cytoplasmic domain interact with TssL. TssL

9 protrudes in the cytoplasm but is anchored by a C-terminal transmembrane helix 10 (TMH). Here, we show that TssL TMH dimerization is required for the stability of the 11 protein and for T6SS function. Using the TOXCAT assay and point mutations of the 23 12 residues of the TssL TMH we identified Thr194 and Trp199 as necessary for TssL TMH 13 dimerization. NMR hydrogen-deuterium exchange experiments demonstrated the 14 existence of a dimer with the presence of Trp185 and Trp199 at the interface. A 15 structural model based on molecular dynamics simulations shows that TssL TMH dimer 16 formation involves $\pi-\pi$ interactions resulting from the packing of the two Trp199 rings 17 at the C-terminus and of the six aromatic rings of Tyr184, Trp185 and Trp188 at the N18 terminus of the TMH. 


\section{Introduction}

3 The Type VI secretion system (T6SS) is a molecular machine widely distributed in

4 proteobacteria and responsible for the contact-dependent secretion of effector proteins into target cells $[1,2]$. Effectors delivered by this apparatus interfere with eukaryotic host cell physiology, or cause damages in bacterial cells [3-6]. The T6SS is therefore involved in

7 pathogenesis and/or bacterial competition. At the molecular level, the T6SS could be represented as a nano-speargun [7-9] (Fig. 1A): an inner tube made of stacked Hcp hexameric rings capped by the VgrG spike complex and wrapped by a contractile sheath is propelled into the target cells during sheath contraction [10-12]. This tubular structure is assembled on a sub-membrane platform - or baseplate - that comprises the TssEFGK and VgrG subunits and is anchored to the cell envelope by the membrane complex [13-18].

In enteroaggregagtive Escherichia coli (EAEC), the T6SS membrane core complex is constituted of the outer membrane-associated TssJ lipoprotein and the TssL and TssM inner membrane proteins $[14,19,20]$ (Fig. 1B and C). TssJ folds as a classical transthyretin with an additional $\alpha$-helix and an extended loop connecting $\beta$-strands 1 and 2 (L1-2) [21-23]. TssM comprises three transmembrane helices $(\mathrm{TMH})$ : TMH1 and TMH2 forms a hairpin located close to the N-terminus, separated from the third helix, TMH3, by a $35-\mathrm{kDa}$ cytoplasmic domain [16,24] (Fig. 1C). The TssM cytoplasmic portion comprises two subdomains with structural homologies to NTPases and the DPY-30 dimerization hairpin [16]. The TssM cytoplasmic domain makes contact with TssL, as well as with TssG and TssK, two components of the tail assembly platform $[13,15,16,18]$. The majority of the EAEC TssM subunit (750 amino-acids) is located in the periplasm and its C-terminal extremity interacts with the TssJ lipoprotein L1-2 loop [14,21]. The EAEC TssL protein is a C-tail membrane protein, anchored to the inner membrane by a C-terminal $\mathrm{TMH}$ that requires the YidC 
1 insertase for its insertion [25,26] (Fig. 1C). The TssL cytoplasmic domain comprises two 3-

2 helix bundles [27-29] and interacts with two subunits of the baseplate, TssE and TssK [15,17].

3 In EAEC, this domain forms weak dimers, but the full-length protein (i.e., comprising the C-

4 terminal transmembrane anchor) is a stable dimer that resists Sodium Dodecyl Sulphate-Poly

5 Acrylamide Gel Electrophoresis (SDS-PAGE; [27]).

6 The TssJLM membrane complex is the first T6SS structure to be assembled. Its

7 biogenesis starts with the positioning of the TssJ lipoprotein and proceeds with the sequential

8 addition of TssM and TssL [14]. Polymerization of this hetero-trimer requires local

9 remodelling of the cell wall layer that is assured by a dedicated peptidoglycan hydrolase or a

10 housekeeping transglycosylase [30,31]. Ten copies of the hetero-trimer polymerize to form

11 the 5-fold symmetry transenvelope channel. The 12- $\AA$ negative-stain electron microscopy structure of the TssJLM complex from enteroaggregative E. coli has been reported [14] (Fig. 1B). It comprises a large base constituted of the cytoplasmic domains and transmembrane segments and a cap composed of TssJ. Base and cap are connected by pillars made of the TssM periplasmic domains [14]. The TssJLM structure has been obtained in a closed conformation and molecular dynamics (MD) simulations have suggested that large conformational modifications of the pillars cause opening of the structure for the passage of the inner tube during sheath contraction [14]. To resist the strength generated during sheath contraction, the TssJLM membrane complex is stably anchored to the cell wall by a peptidoglycan-binding domain either fused at the C-terminus of TssL or carried by an accessory component [20,32].

Although the EM structure of the EAEC TssJLM complex and the X-ray structures of the soluble domains of TssL, TssM and TssJ have been reported, little is known on the transmembrane portion of the complex. It has been shown that the C-terminal anchor of TssL is required for T6SS function and for stable dimerization of TssL [27]. We therefore sought to 
1 gain insights into the structure of the TssL TMH and its dimerization determinants. TOXCAT

2 analyses demonstrated that the TssL TMH is necessary and sufficient for dimerization.

3 Functional assays further showed that TMH-mediated TssL dimerization is required for TssL

4 stability and T6SS function. Finally, scanning site-directed mutagenesis coupled to TOXCAT,

5 structural Nuclear Magnetic Resonance (NMR) studies and MD simulations defined that

6 tryptophan rings engage in $\pi-\pi$ interactions to mediate $\mathrm{Tss} \mathrm{L}^{\mathrm{TM}}$ dimerization.

\section{$8 \quad$ Results}

The TssL transmembrane segment is sufficient for dimerization.

Structure-function analyses of the T6SS TssL cytoplasmic domain revealed that it weakly dimerizes $[17,27])$. By contrast, the full-length protein forms dimers that are stable in SDS-PAGE [27]. We concluded that the C-terminal transmembrane helix strongly contributes to TssL dimerization. To test this hypothesis, we used the TOXCAT assay [33], a two-hybrid assay based on the reconstitution of an active ToxR dimeric regulator upon association of two transmembrane helices. The dimeric ToxR regulator binds the tox promoter and activates the expression of the gene encoding chloramphenicol acetyl-transferase and conferring resistance to chloramphenicol $[33,34]$. The sequence corresponding to the transmembrane helix of TssL $\left(\mathrm{TssL}^{\mathrm{TM}}\right)$ was inserted between the ToxR' domain and the maltose-binding protein (MBP) to yield the ToxR'-TssL ${ }^{\mathrm{TM}}$-MBP fusion protein. Controls include the transmembrane helix of the glycophorin A ( $\mathrm{GpA})$, considered as a model for transmembrane helix association, as well as

a point mutant disrupting dimerization of $\mathrm{GpA}\left(\mathrm{GpA}^{\mathrm{G} 83 \mathrm{I}}\right)$ [33]. Cells producing ToxR'TssL $^{\mathrm{TM}}$-MBP grew on minimal medium with maltose as carbon source (Fig. 2A-B), 24 demonstrating that the MBP localizes in the periplasm and hence that the construct is properly inserted in the membrane via the TssL transmembrane helix. Chloramphenicol disk and 
1 chloramphenicol acetyl-transferase activity (Fig. 2C and 2D) assays further showed that

$2 \mathrm{TssL}^{\mathrm{TM}}$ dimerizes at levels comparable to the GpA control. From these data, we conclude that

$3 \mathrm{TssL}^{\mathrm{TM}}$ dimerizes by itself in the membrane.

\section{$5 \quad$ Thr194 and Trp199 contribute to TssL ${ }^{\mathrm{TM}}$ dimerization.}

To get further information on the driving forces that control TssL $\mathrm{L}^{\mathrm{TM}}$ dimer formation, we used the same TOXCAT approach coupled to site directed mutagenesis screening. A number of motifs involved in transmembrane helix interactions have been reported, such as leucine zipper and GxxGxxxG motifs. Analyses of the TssL ${ }^{\mathrm{TM}}$ sequence reveals the presence of a leucine zipper motif (LxxLxxxL) comprising Leu195, Leu198 and Leu202 (shown in red in Fig. 3A). Leucine zippers are common protein-protein interaction motifs, and such a motif has been shown to be involved in the dimerization of the T4SS-associated VirB10 transmembrane segment [35]. We first engineered mutations to break the $\mathrm{TssL}^{\mathrm{TM}}$ leucine zipper motif by substituting Leu195 and Leu198 by alanines (LALA variant). TOXCAT assays show that mutations of these two leucine residues had only a slight effect on the ability of $\mathrm{TssL}^{\mathrm{TM}}$ to dimerize (Fig. 3B and 3D) and thus contradict the leucine zipper hypothesis. To identify the residues of $\mathrm{TssL}^{\mathrm{TM}}$ involved in dimer formation, we performed a systematic scanning mutagenesis, in which each individual residue has been changed to tryptophan. Tryptophan side-chains are bulky and have been previously shown to disrupt contacts between tightly packed transmembrane segments [36]. Tryptophan residues of TssL ${ }^{\mathrm{TM}}$ were substituted by alanines. TOXCAT assays demonstrated that while a number of substitutions affect the stability of the TssL ${ }^{\mathrm{TM}}$ dimer, only two mutations - Thr194Trp (T194W) and Trp199Ala (W199A) — have a level of chloramphenicol acetyl transferase activity comparable to the absence of TMH (Fig. 3C and 3D). 
Previous data have shown that the TssL transmembrane helix stabilizes the TssL dimer,

2 which then resists SDS-PAGE analysis [27]. We hence tested whether the T194W and

3 W199A substitutions impact the stability of the TssL dimer in SDS-PAGE. As previously

4 observed, the cytoplasmic domain of TssL $\left(\operatorname{TssL}_{\mathrm{C}}\right)$ migrates as a monomer upon SDS-PAGE

5 whereas the dimer of the full-length TssL protein is detected (Fig. 4, lanes 1 and 2

6 respectively). For the T194W and W199A variants of TssL only monomers are observed

7 indicating that the $\mathrm{T} 194 \mathrm{~W}$ and $\mathrm{W} 199 \mathrm{~A}$ mutations have a strong effect on $\mathrm{TssL}^{\mathrm{TM}}$ dimer

8 formation (Fig. 4, lanes 3 and 4).

\section{Structural analysis of the TssL ${ }^{\mathrm{TM}}$ dimer interface}

To investigate the structure of the TssL ${ }^{\mathrm{TM}}$ dimer, we first conducted Nuclear Magnetic Resonance (NMR) experiments using a synthetic peptide corresponding to the TssL transmembrane segment (Met183-Ala207), stabilized by two additional lysine residues at the $\mathrm{N}$ - and C-termini. The TssL ${ }^{\mathrm{TM}}$ peptide was solubilized in presence of SDS because (i) the $\mathrm{TssL}^{\mathrm{TM}}$ dimer has been shown to be stable in SDS-PAGE [27], (ii) in such context, SDS 16 micelles have been previously shown to mimick the membrane environment [37], and (iii) 17 SDS is commonly used for solution NMR analyses of transmembrane domains [38-40]. Proton assignment of the TssL ${ }^{\mathrm{TM}}$ peptide was obtained using TOCSY and NOESY data (Table S1). On the basis of NH/NH nuclear Overhauser effect restraint measurements we concluded that the sequential $\mathrm{NH} / \mathrm{NH}$ distances are in agreement with a structure of the 21 peptide as an extended $\alpha$-helix (Fig. 5A, Fig. S1) [41]. The presence of a unique set of peaks in the NMR spectra suggested that the TssL peptide forms a symmetric dimer in SDS. In this case, it is quite impossible to distinguish intra and intermolecular distance restraints (nOes) at 24 the interface using homonuclear NMR. To investigate the dimer interface, we used hydrogendeuterium (H-D) exchange experiments by recording NOESY experiments in $\mathrm{D}_{2} \mathrm{O}$ buffer. In 
$1 \quad \mathrm{D}_{2} \mathrm{O}$ the $\mathrm{NH}$ groups exposed to the solvent are rapidly converted in ND groups that are not

2 observed in the proton NMR spectra. By contrast, in these conditions, the NH groups

3 protected by a hydrogen bond or by any other contact are slowly converted in ND groups and

4 are still observed in the proton NMR spectra. Fig. 5B and 5C show that the Trp185 and

5 Trp199 ring NHs are significantly more protected to the solvent exchange compared to the

6 amide groups of the rest of the TssL ${ }^{\mathrm{TM}}$ peptide, likely because of their location at the dimer

7 interface. Thus, the H-D exchange experiments support the conclusion of the TOXCAT assay

8 that $\mathrm{TssL}^{\mathrm{TM}}$ dimer interface involves the Trp199 rings at the periplasmic side of the TMH and

9 in addition, provides support to the location of the Trp185 rings at the interface at the

10 cytoplasmic side of the TMH (Fig. 5D). However, rapid H-D exchange was observed for the

11 amide group of Thr194, suggesting that it is not protected and thus that Thr194 is not located

12 at the dimer interface. However, although the NMR approach demonstrated that $\mathrm{TssL}^{\mathrm{TM}}$ has

13 an helical structure and is organized as a dimer in SDS with Trp185 and Trp199 at the

14 interface, it was not possible to define the structure of $\mathrm{TssL}^{\mathrm{TM}}$ using classical approaches of

15 homonuclear NMR.

16 Toward the aim to obtain a structural model of the $\operatorname{TssL}^{\mathrm{TM}}$ dimer and to better define the

17 dimerization interface, we used molecular dynamics (MD) simulations. For comparison, these

18 MD simulations were conducted in conditions identical to the NMR experiments (at 300K, in

19 SDS micelle). First, a 50-ns simulation of the monomer peptide was performed in a SDS

20 micelle containing 110 detergent molecules to generate the basic element, i.e., the TssL ${ }^{\mathrm{TM}}$

21 monomer. Consistent with the NMR data, the averaged structure demonstrated that the

$22 \mathrm{TssL}^{\mathrm{TM}}$ monomer is a straight $\alpha$-helix in SDS environment (Fig. 5E and 6A). This structure

23 further shows that Trp199 and Trp185 belong to the same helix face (Fig. 5E). By contrast,

24 Thr194 and Trp199 belong to opposite faces (Fig. 5E and 6A), and hence, it is unlikely that

25 both residues participate to a homodimeric interaction without substantial structure 
1 disturbance. To explore if such distortions could arise in the context of the dimeric structure,

2 we performed MD simulations of $\mathrm{TssL}^{\mathrm{TM}}$ in the SDS micelle (i.e., in conditions similar to the

3 NMR and SDS-PAGE experiments, in which the TssL ${ }^{\mathrm{TM}}$ dimer was observed). Starting from

4 eight different symmetric conformations (see methods), the superimposition of the eight 5 averaged structures (Fig. 6A) shows no significant changes of the structure of TssL ${ }^{\mathrm{TM}}$. Fig.

$6 \mathrm{6B}$ and $6 \mathrm{C}$ capture the proximity between the Thr194 or Trp199 in each dimer during the 7 course of the 8 simulations (numbered from $0^{\circ}$ to $350^{\circ}$ according to the relative orientation of

8 the helix faces). Interestingly, only the $250^{\circ}$ simulation allows a tight proximity between the

9 Thr194 of the two monomers (Fig. 6B), whereas the $0^{\circ}, 50^{\circ}$ and $350^{\circ}$ simulations bring the 10 Trp199 of both monomers at close proximity (Fig. 6C). These simulations also indicate that 11 the Trp199 of one monomer is never close to the Thr194 residue of the other monomer. As suggested by the organization of residues on the face of the straight $\alpha$-helix (Fig. 5E), the

13 positioning of $\operatorname{Trp} 199$ at the interface also bring aromatic residues of the $\mathrm{TssL}^{\mathrm{TM}} \mathrm{N}$-terminal 14 region (Tyr184, Trp185, Trp188) in close proximity. To further address the role of the 15 aromatic residues at the interface, the ' 0 ' and $350^{\circ}$ simulations were prolonged for $100 \mathrm{~ns}$ (Fig. $6 \mathrm{D}$ and $6 \mathrm{E})$. The two simulations converged to a very similar structure in which eight aromatic rings (Tyr184, Trp185, Trp188, Trp199 from each monomer) interact at the interface of the TMH dimer (Fig. 5F). Interestingly, Trp185 was protected in NMR H-D exchange experiments and thus described as an interfacial residue. Hence, modeling of the $\mathrm{TssL}^{\mathrm{TM}}$ dimer confirms that (i) Thr194 and Trp199 are not located on the same interface, and (ii) that 21 Trp199 likely contributes, as well as residues Tyr184, Trp185 and Trp188, to TssL ${ }^{\mathrm{TM}}$ dimerization (Fig. 5F). The implication of Trp199 to $\mathrm{TssL}^{\mathrm{TM}}$ dimerization during $\mathrm{MD}$ simulations validates the results of the TOXCAT assay and NMR H-D exchange experiments.

24 By contrast to the TOXCAT assay, the NMR and MD data did not highlight a role for residue 25 Thr194. To test whether the replacement of Thr194 by a Trp residue interfere with TssL ${ }^{\mathrm{TM}}$ 
1 dimerization, we performed MD simulations using the $\mathrm{TssL}^{\mathrm{TM}}$ peptide bearing a $\operatorname{Trp}$ at

2 position 194 (Fig. S2). This substitution significantly affects the contact map calculated for

3 the $\mathrm{TssL}^{\mathrm{TM}}$ dimer during a 50-ns simulation. Notably, the contacts that initially involved the

4 N-terminal aromatic cluster decrease (see for example Trp188) to the advantage of new

5 contacts that are formed on the C-terminal region of the peptide (Fig. S2A). From the initial

6 position (in red), this drift leads to a more open conformation (in blue) that moves forward the

7 two N-terminal extremities (Fig. S2B). This structural change corresponds to a huge rise in

8 the root-mean square deviation ( $\mathrm{rmsd}$ ) of monomer $\mathrm{B}$ relative to monomer $\mathrm{A}$, that reaches a

9 plateau at $\sim 5-6 \AA$ in less than $5 \mathrm{~ns}$, increasing to $10 \AA$ after $20 \mathrm{~ns}$, a behavior typical of a loss

10 of structural stability (Fig. S2C). Hence, the lack of interaction between TssL ${ }^{\mathrm{TM}}$ T194W

11 variants in the TOXCAT assay might be correlated to a significant impairment of the $\mathrm{TssL}^{\mathrm{TM}}$

12 dimer integrity that could arise from subtle conformational or dynamic alterations.

14 Transmembrane-mediated TssL dimerization is required for T6SS function.

15 The T6SS assembles a tail-like structure comprising the tail tube made of Hcp hexameric 16 rings wrapped by the sheath. Contraction of the sheath is proposed to propel the Hcp tube 17 toward the target cell. The presence of the Hcp protein in the culture supernatant is therefore a reporter of T6SS activity [42]. Indeed, Hcp is detected in the culture supernatant of a wild-

19 type strain, whereas is absent in the culture supernatant of a $\Delta t s s L$ mutant strain (Fig. 7A).

20 The production of a plasmid-borne full-length TssL protein restores the release of Hcp. By

21 contrast, despite the observation that the W199A variant is produced at levels comparable to

22 wild-type TssL, this mutation abolishes Hcp release (Fig. 7A). The EAEC T6SS has anti-

23 bacterial activity and has been shown to provide a competitive advantage against $E$. coli K-12

24 cells [43]. The results of the competition assays showed that cells producing the W199A TssL

25 mutant are unable to eliminate competitor E. coli K-12 cells (Fig. 7B). 
2 the TssL W199A mutation prevents proper function of the Type VI secretion apparatus in 3 EAEC, suggesting that T6SS function requires dimerization of the TssL transmembrane 4 anchor.

6 TssL dimerization increases TssL stability.

To gain further insights onto the role of $\mathrm{TssL}^{\mathrm{TM}}$ dimerization during $\mathrm{T} 6 \mathrm{SS}$ assembly,

8 we tested whether disrupting TssL ${ }^{\mathrm{TM}}$ dimer formation impact the stability of the full-length

9 TssL. EAEC cells producing TssL or TssL-W199A were subjected to protein synthesis arrest 10 using the ribosomal 30S-specific spectinomycin antibiotic, and steady-state TssL levels were 11 monitored over time. Fig. 8 shows that TssL is a rather stable protein with a half-life of $\sim 50$ min. The W199A substitution causes an important decrease of TssL stability, with a measured half-time $<10 \mathrm{~min}$.

\section{Ligand-induced dimerization of TssL}

Our in vivo results, monitored in the context of the fully assembled T6SS, cannot exclude that

17 the W199A substitution affects other functions or interactions in addition to $\mathrm{TssL}^{\mathrm{TM}}$ dimerization. We hence engineered TssL variants in which the genetically modified FK506binding protein (FKBP), FV, was fused to W199A TssL (Fig. 9A). FKBP are soluble signaling

20 proteins that dimerize in response to a chemical inducer, rapamycin [44]. Hence, the couple $21 \mathrm{~F}_{\mathrm{V}}$ and AP20187, a non-toxic analogue of rapamycin, could be used for ligand-induced 22 dimerization [44,45] (Fig. 9A). $\mathrm{F}_{\mathrm{V}}$ was fused to the TssL C-terminus (Fig. 9A), which is 23 located in the periplasm and permissive to insertion, as TssL subunits fused to peptidoglycan24 binding motifs are commonly found associated with T6SS [32]. TOXCAT assay showed that 25 the presence of AP20187 in the medium overcomes the dimerization defect caused by the 
$1 \mathrm{TssL}^{\mathrm{TM}}$ W199A mutation (Fig. 9B). Ligand-induced dimerization of the TssL W199A variant

2 restores T6SS-mediated Hcp release (Fig. 9C) and antibacterial activity (Fig. 9D), and

3 increases TssL W199A stability to wild-type levels (Fig. 9E-G). Taken together, these results

4 demonstrate that $\operatorname{Trp} 199$ is a key residue for $\mathrm{TssL}^{\mathrm{TM}}$ dimerization and that $\mathrm{TssL}^{\mathrm{TM}}$

5 dimerization is essential for T6SS activity.

7 Discussion

In this study, we report that the TssL transmembrane segment is a major determinant

9 of TssL dimerization. Using a combination of TOXCAT, NMR and MD assays, we show that

$10 \mathrm{TssL}^{\mathrm{TM}}$ dimer formation is mediated by $\pi-\pi$ interactions involving three aromatic residues at

11 the cytoplasmic side (Tyr184, Trp185 and Trp188) and one tryptophan at the periplasmic side

12 (Trp199). We further provide evidence for a critical role of TM-mediated TssL dimerization

13 in T6SS stability and activity.

14 Few data are available in the field of bacterial secretion systems regarding the role of

15 TM segments, and more specifically how interactions between TM helices participate to the 16 assembly of these multiprotein complexes. However, the dimerization of the Agrobacterium 17 tumefaciens VirB10 protein TM helix is required for Type IV secretion $[35,46]$.

18 Our results highlighted the specific and important role of $\mathrm{TssL}^{\mathrm{TM}}$ aromatic residues in

19 dimer formation. Interestingly, $\mathrm{TssL}^{\mathrm{TM}}$ dimerization is mediated by a total of eight aromatic 20 rings, with six of them forming a tight patch. $\pi-\pi$ packing of aromatic rings is a common 21 interaction motif in proteins $[47,48]$, but is also a very common interface between proteins 22 and ligands [49]. The involvement of aromatic-aromatic interactions in TM helices is not well 23 documented [50]. By contrast, cation- $\pi$ interactions, such as Lys-Trp interactions, have been 24 shown to control TM interactions within the chloride intracellular channel protein 1 (CLIC1) 25 [51]. Rather, tryptophan side-chains in $\mathrm{TMH}$ have usually functional roles including 
1 selectivity or gatekeepers such as in conjugation coupling proteins [52] and proton or 2 potassium channels [53-56], and locks for rotameric switches, such as for the ghrelin receptor

3 [57]. Interestingly, an alignment of the TMH sequences of T6SS-associated TssL homologues 4 covering the different T6SS subfamilies shows that tryptophan or aromatic residues are highly 5 conserved at the N- and C-termini of the TMHs (Fig. 10). This conservation is also shared 6 with T4bSS-associated IcmH/DotU proteins (Fig. 10), highlighting the importance of 7 aromatic rings at these positions. It is noteworthy that, by contrast to the EAEC TssL TMH, 8 only one aromatic residue is usually conserved at the N-terminus. This observation suggests 9 that the packing between two aromatic rings might be sufficient to stabilize TssL TMH 10 formation at the N-terminus, and thus may explain why single substitution of the Y184, W185 or W188 residue does not significantly impact dimer formation in the TOXCAT assay. It is worthy to note that NMR H-D experiments did not fully copy the results of the TOXCAT assay. However, the MD simulations provided molecular explanations for all the discrepancies. First, no role of residue Thr194 in TssL ${ }^{\mathrm{TM}}$ dimerization could be inferred by HD experiments, whereas the T194W substitution strongly interferes with dimerization using TOXCAT. However, MD simulations using a peptide corresponding to the T194W variant 17 showed that this substitution induces a destabilization of the $\operatorname{TssL}^{\mathrm{TM}}$ dimer packing and a significant motion of one monomer relative to the other. Interestingly, the threonine sidechain tends to bend $\alpha$-helices by the formation of an additional hydrogen bond between its side-chain $\gamma \mathrm{O}$ atom and the i-3 or i-4 peptide carboxyl oxygen [58]. This additional hydrogen bond also alters the dynamic of the helix bending [59] in a way that, in the $\mathrm{TssL}^{\mathrm{TM}}$ peptide, opposes to the formation of a coiled-coil on the dimerization face. Hence, incorporating a small disturbance in bending flexibility at one side of the TMH results in a significant 24 displacement of the residues located at the other side [58], such as we observed in the case of 25 the TssL ${ }^{\mathrm{TM}}$ dimer. In conclusion, although Thr194 does not participate directly to the $\mathrm{TssL}^{\mathrm{TM}}$ 
1 interface, the additional hydrogen bond contributed by the side-chain is important to maintain

2 the $\mathrm{TssL}^{\mathrm{TM}}$ in a conformation competent for dimerization. The quantitative TOX-CAT assays

3 also indicated that several substitutions along the helix affect $\mathrm{Tss}^{\mathrm{TM}}$ dimer formation, albeit

4 to a lower extent compared to T194W. While we did not conduct MD simulations for each

5 variant, it is likely that these substitutions slightly impact $\mathrm{TssL}^{\mathrm{TM}}$ stability and/or

6 conformation, and thus indirectly affect its dimerization. Second, the packing of the six

7 aromatic rings (Tyr184, Trp185 and Trp188) at the N-terminal portion of the TssL $\alpha$-helix is

8 an important determinant of $\mathrm{TssL}^{\mathrm{TM}}$ dimerization, but none of the $\mathrm{Y} 184 \mathrm{~W}, \mathrm{~W} 185 \mathrm{~A}$ and

9 W188A substitutions affects dimerization by TOXCAT. As noted above, the lack of effect of

10 these single substitutions on TOXCAT can be explained by the redundancy of the $\pi-\pi$

11 interactions between these aromatic rings, an hypothesis in agreement with the observation

12 that only one aromatic residue is usually conserved at the $\mathrm{TssL}^{\mathrm{TM}} \mathrm{N}$-termini.

In between the N-terminal Tyr184, Trp185 and Trp188 and the C-terminal Trp199

14 residues of the dimer interface are located the three leucine residues ascertained as a potential leucine zipper. However, the TOXCAT assay showed that mutation of this potential leucine zipper did not impact $\mathrm{TssL}^{\mathrm{TM}}$ dimerization. Interestingly, contrarily to the prototypical motif

17 found in the CGN4 leucine zipper [60], the structure of the TssL ${ }^{\mathrm{TM}}$ peptide revealed that it

18 does not present a coiled-coil structure. As a consequence, the leucine side-chains point 19 toward different directions and hence there is room to accommodate Trp substitutions owing to Trp flexibility due to the free rotation along the $C \alpha-C \beta$ and $C \beta-C \gamma$ bonds.

The development of TssL-F fusions to control TssL dimer levels and hence T6SS assembly is a remarkable tool. Because the assembly of the TssJLM membrane complex represents an early stage of T6SS biogenesis [13,14,61], the ligand-inducible TssL dimerization could be used to synchronize T6SS assembly and activities. 

basis for the development of synthetic peptides that would inhibit TssL dimerization and thus T6SS assembly. Hydrophobic peptides interfering with transmembrane domains have been used to block the action of neuropilin-1, an actor of the human Hedgehog signalling pathway

5 that is involved in development disorders and cancers $[62,63] . \mathrm{TssL}^{\mathrm{TM}}$-targeting peptides that 6 mimick the dimerization interface might be of specific interest to diminish the virulence or the anti-bacterial activity of important bacterial pathogens that use the T6SS to colonize the host, such as Vibrio cholerae, Pseudomonas aeruginosa, or Salmonella enterica [64-68].

\section{Material and Methods}

\section{Bacterial strains, media, growth conditions and chemicals}

The bacterial strains used in this study are listed in Table S2. Escherichia coli K-12 DH5 $\alpha$, W3110 and NT326 [69] were used for cloning procedures, anti-bacterial competition and TOXCAT assays, respectively. The enteroaggregative E. coli strain 17-2 and its $\Delta t s s L$ (previously described as $\Delta s c i P$; [20]) derivative were used for this study. Strains were routinely grown in $\mathrm{LB}$ at $37^{\circ} \mathrm{C}$ with aeration. The TOXCAT assay was performed in M9 minimal medium with glucose or maltose as carbon source. Expression of the T6SS gene cluster was induced in Scil-inducing medium (SIM; M9 minimal medium supplemented with LB $10 \%$, glycerol $0.4 \%$, casaminoacids $40 \mu \mathrm{g} \cdot \mathrm{mL}^{-1}, \mathrm{MgCl}_{2} 2 \mathrm{mM}, \mathrm{CaCl}_{2}$ $0.1 \mathrm{mM}$, Vitamin B1 $200 \mu \mathrm{g} . \mathrm{mL}^{-1}$ [70]). Plasmids and mutations were maintained by the addition of ampicillin $\left(100 \mu \mathrm{g} \cdot \mathrm{mL}^{-1}\right.$ for $E$. coli $\mathrm{K}-12,200 \mu \mathrm{g} \cdot \mathrm{mL}^{-1}$ for EAEC) or kanamycin $\left(50 \mu \mathrm{g} \cdot \mathrm{mL}^{-1}\right.$ for $E$. coli K-12). Protein synthesis was arrested by addition of spectinomycin $\left(200 \mu \mathrm{g} \cdot \mathrm{mL}^{-1}\right)$. Induction of genes cloned into pIBA vectors was obtained with anhydrotetracyclin (AHT - used at $0.02 \mu \mathrm{g} \cdot \mathrm{mL}^{-1}$ throughout the study; IBA Technologies). The AP20187 dimerization inducer was purchased from Clinisciences and used at $20 \mathrm{mM}$. The anti-TolB polyclonal antibodies are from the laboratory 
1 monoclonal antibodies and the goat secondary anti-mouse antibodies coupled to alkaline phosphatase

2 (Jackson Immuno) or AlexaFluor ${ }^{\circledR} 680$ (Molecular Probes) are commercially available.

\section{$4 \quad$ Plasmid construction}

5 The plasmids used in this study are listed in Table S2. Polymerase Chain Reactions (PCR) were 6 performed with a Biometra thermocycler, using the Pfu Turbo DNA polymerase (Stratagene). Custom oligonucleotides were synthesized by Sigma-Aldrich and are listed in Table S2. Plasmids pIBA-TssL and pIBA-TssL $\mathrm{C}_{\mathrm{C}}$ producing the full-length and the cytoplasmic domain (residues 1-183) of TssL protein fused to a N-terminal FLAG sequence have been previously described [25]. The sequence encoding the TssL transmembrane helix has been inserted into the pcckan vector [69] after PCRamplification from the EAEC 17-2 chromosome DNA using primers 5-TssLTM-NheI and 3-TssLTMBamHI. The NheI-BamHI fragment was then inserted into pcckan digested by the same restriction enzymes to yield pcc-TssL-TM. FK506-binding protein (FKBP) fusion chimeras were engineered by a double PCR technique, allowing amplification of the FKBP(F36V) sequence (from plasmid pPSV35CV-ppkA-Fv; [45]) flanked by extensions annealing to the target vector [20,71]. The product of the first PCR has then been used as oligonucleotides for a second PCR using the pIBA-TssL or pccTssL-TM as template. Site-directed mutagenesis was performed using complementary oligonucleotides bearing the desired substitutions. All constructs have been verified by colony PCR, restriction analyses and DNA sequencing (Genome Express).

\section{Antibacterial competition assay}

The antibacterial growth competition assay was performed as previously described [43]. Briefly, the wild-type E. coli strain W3110 bearing the pUA66-rrnB plasmid $\left(\operatorname{Kan}^{\mathrm{R}}[72]\right)$ was used as prey in the competition assay. The pUA66-rrnB plasmid provides a strong constitutive green fluorescent $\left(\mathrm{GFP}^{+}\right)$ phenotype. Attacker and prey cells were grown for $16 \mathrm{~h}$ in SIM medium, and then diluted in SIM to allow maximal expression of the scil gene cluster [70]. Once the culture reached an absorbance at $\lambda=600 \mathrm{~nm}\left(A_{600}\right)$ of 0.8 , cells were harvested and normalized to $A_{600}=10$ in SIM. Attacker and prey cells were mixed to a 4:1 ratio and $20-\mu \mathrm{L}$ drops of the mixture were spotted in triplicate onto a pre- 
1 warmed dry SIM agar plate supplemented with $0.02 \mu \mathrm{g} \cdot \mathrm{mL}^{-1}$ of AHT. After 4-hour incubation at $37^{\circ} \mathrm{C}$,

2 fluorescent images were recorded with a LI-COR Odyssey imager. The bacterial spots were scratched

3 off, and cells were resuspended in LB medium and normalized to an $A_{600}=0.5$. Triplicates of $200 \mu \mathrm{L}$

4 were transferred into wells of a black 96-well plate (Greiner) and the $A_{600}$ and fluorescence (excitation,

$5485 \mathrm{~nm}$; emission, $530 \mathrm{~nm}$ ) were measured with a Tecan Infinite M200 microplate reader. The relative

6 fluorescence was expressed as the intensity of fluorescence divided by the $A_{600}$, after subtracting the

7 values of a blank sample. For estimating bacterial prey survival, colony-forming units (cfu) were

8 enumerated after plating serial dilutions on LB plates supplemented with kanamycin. The experiments

9 were done in triplicate, with identical results, and we report here the results of a representative

10 experiment.

\section{Hcp release assay}

13 Supernatant and cell fractions have been separated as previously described [20] except that cells were grown in SIM medium. When necessary, AHT was added 30 minutes before harvesting the cells. Briefly, $2 \times 10^{9}$ cells producing HA epitope-tagged Hcp (from plasmid pOK-Hcp $\mathrm{HA}$; [20]) were harvested and collected by centrifugation at $2,000 \times g$ for $5 \mathrm{~min}$. The supernatant fraction was then subjected to a second low-speed centrifugation, a high-speed centrifugation $(16,000 \times g$ for $15 \mathrm{~min})$ and filtered on sterile polyester membranes with a pore size of $0.2 \mu \mathrm{m}$ (membrex 25 PET, membraPure $\mathrm{GmbH}$ ) before precipitation with trichloroacetic acid (TCA) 15\%. Cells and precipitated supernatant were resuspended in loading buffer and analysed by SDS-PAGE and immunoblotting with the anti-HA antibody. As control for cell lysis, Western blots were probed with antibodies raised against the periplasmic TolB protein.

\section{TOXCAT assay}

The TOXCAT assay was performed as described [33,34,69]. MalE complementation assay: NT326 cells producing the TOXCAT fusion were streaked onto M9 minimal medium plates supplemented with $0.4 \%$ glucose or with $0.4 \%$ maltose as the sole carbon source. Plates were incubated for 2 days at 
$1 \mathrm{~mm}$ filter disk (Bio-Rad, $0.2 \mu \mathrm{m}$ pore size) disposed onto a sterile empty petri dish. After drying, the

2 filter was deposited at the center of a dried LB plate supplemented with ampicillin. Plates were 3 incubated for 6 hours at $37^{\circ} \mathrm{C}$ before removing the filter disk. $2 \mathrm{~mL}$ of a culture of NT326 cells

4 producing the TOXCAT fusion were used to make a lawn and plates were incubated $\mathrm{ON}$ at $37^{\circ} \mathrm{C}$. The experiments have been performed in triplicate. TOXCAT quantitative assay: NT326 cells producing the TOXCAT fusion were inoculated into $10 \mathrm{~mL}$ of $\mathrm{LB}$ supplemented with ampicillin and grown to an $A_{600}=1$ at $37^{\circ} \mathrm{C}$ with vigorous shaking. $10^{10}$ cells ( $\sim 6 A_{600}$ units) were harvested by centrifugation and washed with $1 \mathrm{~mL}$ of sonication buffer ( $25 \mathrm{mM}$ Tris-HCl, $2 \mathrm{mM}$ EDTA, pH8.0). Cells were then resuspended in $1 \mathrm{~mL}$ of sonication buffer and lysed by sonication. CAT activity measurements: $12 \mu \mathrm{L}$ of lysate were mixed with $300 \mu \mathrm{L}$ of reaction buffer $\left(0.1 \mathrm{mM}\right.$ acetyl-CoA, $0.4 \mathrm{mg} \cdot \mathrm{mL}^{-1}$ 5,5'-dithiobis(2-nitrobenzoic acid), 0.1 M Tris- $\mathrm{HCl}, \mathrm{pH} 7.8$ ), and the absorbance was acquired at $\lambda=412 \mathrm{~nm}$ at 10 sec intervals for a minimum of 4 min to measure the background rate of acetyl-CoA hydrolysis. Then, $12 \mu \mathrm{L}$ of $2.5 \mathrm{mM}$ chloramphenicol was added, and the absorbance was measured every $10 \mathrm{sec}$. for 1 min. Each lysate was assayed in triplicate, and the slopes of the recorded graphs were converted to enzyme activity units $(\varepsilon=13,600$ at $\lambda=412 \mathrm{~nm})$.

\section{AP20187-mediated FK506-binding protein dimerization}

Dimerization of FKBP(F36V) was induced by the addition of $20 \mathrm{mM}$ AP20187 (Clinisciences, resuspended in dimethylsulfoxide) in the culture medium.

\section{Protein stability assay}

Cultures were grown at $37^{\circ} \mathrm{C}$ to an $A_{600}=0.8$, and $200 \mu \mathrm{g} \cdot \mathrm{mL}^{-1}$ of spectinomycin was added to stop protein synthesis [73]. A sample was harvested at time zero and equivalent samples were harvested 5, $10,15,30,60,120,240$ and $480 \mathrm{~min}$. after spectinomycin addition. The bacterial density was measured at different time points to verify that no growth occurred. Cell pellets were resuspended in Laemmli loading buffer, heated for $15 \mathrm{~min}$ at $96^{\circ} \mathrm{C}$, and subjected to $12.5 \%$ SDS-PAGE. Western- 
1 images were recorded at $\lambda=700 \mathrm{~nm}$ using an LI-COR Odyssey imager. Image analyses were

2 performed with the ImageJ processing program using the Fiji interface [74]. The image was first

3 converted to grayscale in .jpg format. The rectangle tool of ImageJ was used to select a rectangular

4 area of the size corresponding to the lane width, in order to cover the minimal area to contain the

5 whole of the largest band. The same frame was used to select each TssL band of the nitrocellulose

6 membrane. For each selection, the number of pixels was calculated. A control region with no band

7 was also selected to subtract the background. The number of pixels of each band, relative to time 0

8 was then plotted against time. The degradation rate constant $(\mathrm{k})$ was calculated as the slope of the

9 linear curve obtained by plotting the Napierian logarithm of each intensity value against time and

10 fitting to a first-order decay function. Half-life values $\left(\tau_{1 / 2}\right)$ were obtained using the equation $\tau_{1 / 2}=$ $11 \ln (2) / \mathrm{k}$

12

13 TssL $^{\mathrm{TM}}$ peptide synthesis and structural analysis by NMR

14 A synthetic peptide, corresponding to the TssL transmembrane segment (residues Met183-Ala207) with two additional lysine residues at the $\mathrm{N}$ - and C-terminus of the peptide (sequence: KKMYWLSWGAGIVTLAGLWCVLSSVLAKK) was synthesized by Schafer-N (Danemark). Two samples were prepared, one in $\mathrm{H}_{2} \mathrm{O}$ and one in $\mathrm{D}_{2} \mathrm{O}$ for proton/deuterium exchange experiments. For each sample $2 \mathrm{mg}$ of peptide were solubilized in $500 \mu \mathrm{L}$ of D25-Sodium Dodecyl Sulfate $1 \%$, in 10 $\mathrm{mM} \mathrm{KH}_{2} \mathrm{PO} 4,1 \mathrm{mM}$ D10-Dithiothreitol, $\mathrm{pH}$ 4.5. TOCSY and NOESY experiments were recorded at $300 \mathrm{~K}$ using a Bruker Avance III 600 spectrometer equipped with a TCI cryoprobe. Mixing time for the NOESY experiments was $300 \mathrm{~ms}$. The NMR spectra were processed with Topsin software (Bruker) and proton assignment was obtained using the CARA software [75].

\section{Molecular dynamics simulations}

25 On the basis of NMR data, the sequence corresponding to the synthetic TssL peptide was modelled as 26 a canonical $\alpha$-helix using the Deepview software [76]. The structure was duplicated using VMD and a 27 Tcl script was used to build 8 symmetric dimer conformations by translating and rotating monomers. 
1 The resulting structures were in close contact and in parallel arrangements. A peptide-detergent 2 complex was built using the Charmm-gui server [77]. The resulting micelles contained 112 SDS 3 molecules and 17,600 water molecules. The system was neutralized using $112 \mathrm{Na}^{+}$and $8 \mathrm{Cl}^{-}$ions. All

4 the simulations were performed using Gromacs 5.1 with the Charmm 36 forcefield. For the 5 simulations, the default protocol and MD parameters generated by Charmm-gui were conserved.

6 Long range electrostatic forces were computed above $1.2 \mathrm{~nm}$, and a switching function was used for 7 short-range electrostatics forces between 1 and $1.2 \mathrm{~nm}$. The system was minimized using the steepest 8 descent algorithm until the maximum force converges under $1000 \mathrm{~kJ} / \mathrm{mol} / \mathrm{nm}$. The micelle and the 9 water environment around the peptide were relaxed using several rounds of simulations starting from 10 constrained peptide, then relaxing forces on side-chains and finally on the main chain. The system was 11 thermalized and equilibrated at $300 \mathrm{~K}$ using several rounds of simulation over 375 ps. A timestep of 1 12 fs, with the LINCS algorithm applied to constraint bonds that involves $\mathrm{H}$ atoms. The temperature was 13 controlled using a multi-coupling scheme separating the peptide and the micelle, with an isotropic 14 Berendsen thermostat and a coupling time constant of $5 \mathrm{ps}$ and $1 \mathrm{ps}$ for heating and equilibration 15 respectively. For the production, a coupling scheme with 10-ns trajectories was produced for the 16 various systems. The simulations were filtered on the basis of MD analysis, TOXCAT and NMR data 17 that resulted on the selection of two simulations that were extended to 100 ns. Finally, simulations of the $\mathrm{T} 194 \mathrm{~W}$ variant were started from an equilibrated conformation of the wild-type peptide. MD

19 simulations of Thr194 variants were performed after substitution of Thr194 by Trp on the TssL ${ }^{\mathrm{TM}}$ 20 structure using the Depview software for 20 ns respectively.

\section{Acknowledgements}

23 We thank Olivier Bornet and the NMR facilities of the Institut de Microbiologie de la 24 Mediterranée for technical assistance in recording NMR experiments, Joseph Mougous 25 (University of Washington, Seattle, USA) for providing the FKBP (F36V)-encoding plasmid 
1 pPSV35CV-ppkA-Fv, Pierre Hubert, James Sturgis and members of the Cascales, Lloubès,

2 Bouveret, Sturgis and Cambillau research groups for discussions, Molly Ba, Isabelle Bringer,

3 Annick Brun, and Oliver Uderso for technical assistance, Matt Edessin for encouragements,

4 and the four anonymous referees for their constructive comments and suggestions. This work

5 was supported by the Aix-Marseille Université (AMU), the Centre National de la Recherche

6 Scientifique (CNRS), and grants from the Agence Nationale de la Recherche (ANR-14-CE14-

7 0006-02) and the European Society for Clinical Microbiology and Infectious Diseases

8 (ESCMID) to E.C. and E.D., respectively. The MD simulations were performed using the

9 high performance computing (HPC) resources from the Grand Equipement National de Calcul

10 Intensif of the Centre Informatique National de l'Enseignement Supérieur (GENCI-CINES

11 grant 2016077044). A.Z. was supported by a doctoral fellowship from the French Ministère

12 de la Recherche and a Fondation pour la Recherche Médicale end-of-thesis fellowship

13 (FDT20140931060). E.D. is supported by the Institut National de la Santé et de la Recherche

14 Médicale (INSERM).

\section{References}

17 [1] F. Boyer, G. Fichant, J. Berthod, Y. Vandenbrouck, I. Attree, Dissecting the bacterial type VI secretion system by a genome wide in silico analysis: what can be learned from available microbial genomic resources?, BMC Genomics 10 (2009) 104.

[2] J.M. Silverman, Y.R. Brunet, E. Cascales, J.D. Mougous, Structure and regulation of the type VI secretion system, Ann. Rev. Microbiol. 66 (2012) 453-472.

[3] A.B. Russell, S.B. Peterson, J.D. Mougous, Type VI secretion system effectors: poisons with a purpose, Nat. Rev. Microbiol. 12 (2014) 137-148. 
1 [4] E. Durand, C. Cambillau, E. Cascales, L. Journet, VgrG, Tae, Tle, and beyond: the versatile arsenal of type VI secretion effectors, Trends Microbiol. 22 (2014) 498-507.

[5] F.R. Cianfanelli, L. Monlezun, S.J. Coulthurst, Aim, load, fire: the type VI secretion system, a bacterial nanoweapon, Trends Microbiol. 24 (2016) 51-62.

[6] A. Hachani, T.E. Wood, A. Filloux, Type VI secretion and antihost effectors, Curr. Opin. Microbiol. 29 (2015) 81-93.

[7] A. Zoued, Y.R. Brunet, E. Durand, M.S. Aschtgen, L. Logger, B. Douzi, L. Journet, C. Cambillau, E. Cascales, Architecture and assembly of the type VI secretion system, Biochim. Biophys. Acta 1843 (2014) 1664-1673.

[8] B.T. Ho, T.G. Dong, J.J. Mekalanos, A view to a kill: the bacterial Type VI secretion system, Cell Host Microbe 15 (2014) 9-21.

[9] M. Basler, Type VI secretion system: secretion by a contractile nanomachine, Philos. Trans. R. Soc. Lond. Ser. B Biol. Sci. 370 (2015) 1679.

[10] M. Basler, M. Pilhofer, G.P. Henderson, G.J. Jensen, J.J. Mekalanos, Type VI secretion requires a dynamic contractile phage tail-like structure, Nature 483 (2012) 182-186.

[11] M. Basler, B.T. Ho, J.J. Mekalanos, Tit-for-tat: type VI secretion system counterattack during bacterial cell-cell interactions, Cell 152 (2013) 884-894.

[12] Y.R. Brunet, L. Espinosa, S. Harchouni, T. Mignot, E. Cascales, Imaging type VI secretion-mediated bacterial killing, Cell Rep. 3 (2013) 36-41.

[13] Y.R. Brunet, A. Zoued, F. Boyer, B. Douzi, E. Cascales, The type VI secretion TssEFGK-VgrG phage-like baseplate is recruited to the TssJLM membrane complex via multiple contacts and serves as assembly platform for tail tube/sheath polymerization, PLoS Genet. 15 (3) (2015) e1005545.

[14] E. Durand, V.S. Nguyen, A. Zoued, L. Logger, G. Péhau-Arnaudet, M.S. Aschtgen, S. Spinelli, A. Desmyter, B. Bardiaux, A. Dujeancourt, A. Roussel, C. Cambillau, E. Cascales, R. Fronzes, Biogenesis and structure of a type VI secretion membrane core complex, Nature 523 (2015) 555-560. 
1 [15] A. Zoued, E. Durand, C. Bebeacua, Y.R. Brunet, B. Douzi, C. Cambillau, E. Cascales, L. Journet, TssK is a trimeric cytoplasmic protein interacting with components of both phage-like and membrane anchoring complexes of the Type VI secretion system, J. Biol. Chem. 288 (2013) 27031-27041.

[16] L. Logger, M.S. Aschtgen, M. Guérin, E. Cascales, E. Durand, Molecular dissection of the interface between the Type VI secretion TssM cytoplasmic domain and the TssG baseplate component, J Mol Biol. 428 (2016) 4424-4437.

[17] A. Zoued, C.J. Cassaro, E. Durand, B. Douzi, A.P. España, C. Cambillau, L. Journet, E. Cascales, Structure-function analysis of the TssL cytoplasmic domain reveals a new interaction between the Type VI secretion baseplate and membrane complexes, J Mol Biol. 428 (2016) 4413-4423.

[18] V.S. Nguyen, L. Logger, S. Spinelli, P. Legrand, T.T. Huyen Pham, T.T. Nhung Trinh, Y. Cherrak, A. Zoued, A. Desmyter, E. Durand, A. Roussel, C. Kellenberger, E. Cascales, C. Cambillau, Type VI secretion TssK baseplate protein exhibits structural similarity with phage receptor-binding proteins and evolved to bind the membrane complex, Nat Microbiol 2 (2017) 17103.

[19] M.S. Aschtgen, C.S. Bernard, S. De Bentzmann, R. Lloubès, E. Cascales, SciN is an outer membrane lipoprotein required for type VI secretion in enteroaggregative Escherichia coli, J. Bacteriol. 190 (2008) 7523-7531.

[20] M.S. Aschtgen, M. Gavioli, A. Dessen, R. Lloubès, E. Cascales, The SciZ protein anchors the enteroaggregative Escherichia coli type VI secretion system to the cell wall, Mol. Microbiol. 75 (2010) 886-899.

[21] C. Felisberto-Rodrigues, E. Durand, M.S. Aschtgen, S. Blangy, M. Ortiz-Lombardia, B. Douzi, C. Cambillau, E. Cascales, Towards a structural comprehension of bacterial type VI secretion systems: characterization of the TssJ-TssM complex of an Escherichia coli pathovar, PLoS Pathogens. 7 (2011) e1002386.

[22] V.A. Rao, S.M. Shepherd, G. English, S.J. Coulthurst, W.N. Hunter, The structure of Serratia marcescens Lip, a membrane-bound component of the type VI secretion system, Acta Crystallogr D Biol Crystallogr. 67 (2011) 1065-1072. 
1 [23] C.S. Robb, M. Assmus, F.E. Nano, A.B. Boraston, Structure of the T6SS lipoprotein 2 TssJ1 from Pseudomonas aeruginosa. Acta Crystallogr Sect F Struct Biol Cryst Commun. 69 (2013) 607-610.

[24] L.S. Ma, J.S. Lin, E.M. Lai, An IcmF family protein, ImpLM, is an integral inner membrane protein interacting with ImpKL, and its walker a motif is required for type VI secretion systemmediated hcp secretion in Agrobacterium tumefaciens, J. Bacteriol. 191 (2009) 4316-4129.

[25] M.S. Aschtgen, A. Zoued, R. Lloubès, L. Journet, E. Cascales, The C-tail anchored TssL subunit, an essential protein of the enteroaggregative Escherichia coli Sci-1 type VI secretion system, is inserted by YidC, Microbiologyopen 1 (2012) 71-82.

[26] E. Pross, L. Soussoula, I. Seitl, D. Lupo, A. Kuhn, Membrane targeting and insertion of the C-tail protein SciP, J Mol Biol. 428 (2016) 4218-4227.

[27] E. Durand, A. Zoued, S. Spinelli, P.J. Watson, M.S. Aschtgen, L. Journet, C. Cambillau, E. Cascales, Structural characterization and oligomerization of the TssL protein, a component shared by bacterial type VI and type IVb secretion systems, J. Biol. Chem. 287 (2012) 14,157-14,168.

[28] C.S. Robb, F.E. Nano, A.B. Boraston, The structure of the conserved type six secretion protein TssL (DotU) from Francisella novicida, J Mol Biol. 419 (2012) 277-283.

[29] J.H. Chang, Y.G. Kim, Crystal structure of the bacterial type VI secretion system component TssL from Vibrio cholerae, J Microbiol. 53 (2015) 32-37.

[30] B.S. Weber, S.W. Hennon, M.S. Wright, N.E. Scott, V. de Berardinis, L.J. Foster, J.A. Ayala, M.D. Adams, M.F. Feldman, Genetic dissection of the Type VI secretion system in Acinetobacter and identification of a novel peptidoglycan hydrolase, TagX, required for its biogenesis, MBio. 7 (2016) e01253-16.

[31] Y.G. Santin, E. Cascales, Domestication of a housekeeping transglycosylase for assembly of a Type VI secretion system, EMBO Rep. 18 (2017) 138-149.

[32] M.S. Aschtgen, M.S. Thomas, E. Cascales, Anchoring the type VI secretion system to the peptidoglycan: TssL, TagL, TagP... what else?, Virulence. 1 (2010) 535-540. 
1 [33] W.P. Russ, D.M. Engelman, TOXCAT: a measure of transmembrane helix association in a biological membrane, Proc Natl Acad Sci U S A. 96 (1999) 863-868.

[34] L. Logger, A. Zoued, E. Cascales, Fusion reporter approaches to monitoring transmembrane helix interactions in bacterial membranes, Methods Mol Biol. 1615 (2017) 199-210.

[35] J. Garza, P.J. Christie, A putative transmembrane leucine zipper of Agrobacterium VirB10 is essential for T-pilus biogenesis but not type IV secretion, J Bacteriol. 195 (2013) 3022-3034.

[36] L.L. Sharp, J. Zhou, D.F. Blair, Features of MotA proton channel structure revealed by tryptophan-scanning mutagenesis, Proc Natl Acad Sci U S A. 92 (1995) 7946-7950.

[37] D.V. Tulumello, C.M. Deber, SDS micelles as a membrane-mimetic environment for transmembrane segments, Biochemistry. 48 (2009) 12096-12103.

[38] J. Venkatraman, G.A. Nagana Gowda, P. Balaram, Structural analysis of synthetic peptide fragments from EmrE, a multidrug resistance protein, in a membrane-mimetic environment, Biochemistry. 41 (2002) 6631-6639.

[39] Y. Wu, S.C. Shih, N.K. Goto, Probing the structure of the Ff bacteriophage major coat protein transmembrane helix dimer by solution NMR, Biochim Biophys Acta. 1768 (2007) 3206-3215.

[40] J.H. Chill, J.M. Louis, C. Miller, A. Bax, NMR study of the tetrameric KcsA potassium channel in detergent micelles, Protein Sci. 15 (2006) 684-698.

[41] K. Wuthrich, NMR of proteins and nucleic acids, Ed. Wiley (1986)

[42] E. Cascales, The type VI secretion toolkit, EMBO Rep. 9 (2008) 735-741.

[43] N. Flaugnatti, T.T. Le, S. Canaan, M.S. Aschtgen, V.S. Nguyen, S. Blangy, C. Kellenberger, A. Roussel, C. Cambillau, E. Cascales, L. Journet, A phospholipase A1 anti-bacterial T6SS effector interacts directly with the C-terminal domain of the VgrG spike protein for delivery. Mol Microbiol. 99 (2016) 1099-1118. 
1 [44] T.W. Corson, N. Aberle, C.M. Crews, Design and applications of bifunctional small molecules: why two heads are better than one, ACS Chem Biol. 3 (2008) 677-692.

[45] F. Hsu, S. Schwarz, J.D. Mougous, TagR promotes PpkA-catalysed type VI secretion activation in Pseudomonas aeruginosa, Mol Microbiol. 72 (2009) 1111-1125.

[46] S.J. Jakubowski, J.E. Kerr, I. Garza, V. Krishnamoorthy, R. Bayliss, G. Waksman, P.J. Christie, Agrobacterium VirB10 domain requirements for type IV secretion and T pilus biogenesis, Mol Microbiol. 71 (2009) 779-794.

[47] R. Anjana, M.K. Vaishnavi, D. Sherlin, S.P. Kumar, K. Naveen, P.S. Kanth, K. Sekar, Aromatic-aromatic interactions in structures of proteins and protein-DNA complexes: a study based on orientation and distance, Bioinformation. 8 (2012) 1220-1224.

[48] D.E. Robillard, P.T. Mpangase, S. Hazelhurst, F. Dehne, SpeeDB: fast structural protein searches, Bioinformatics. 31 (2015) 3027-3034.

[49] S. Li, Y. Xu, Q. Shen, X. Liu, J. Lu, Y. Chen, T. Lu, C. Luo, X. Luo, M. Zheng, H. Jiang, Non-covalent interactions with aromatic rings: current understanding and implications for rational drug design, Curr Pharm Des. 19 (2013) 6522-6533.

[50] R.M. Johnson, K. Hecht, C.M. Deber, Aromatic and cation-pi interactions enhance helixhelix association in a membrane environment, Biochemistry. 46 (2007) 9208-9214.

[51] B. Peter, A.A. Polyansky, S. Fanucchi, H.W. Dirr, A Lys-Trp cation- $\pi$ interaction mediates the dimerization and function of the chloride intracellular channel protein 1 transmembrane domain, Biochemistry. 53 (2014) 57-67.

[52] I. Tato, S. Zunzunegui, F. de la Cruz, E. Cabezon, TrwB, the coupling protein involved in DNA transport during bacterial conjugation, is a DNA-dependent ATPase, Proc Natl Acad Sci U S A. 102 (2005) 8156-8161.

[53] J.K. Williams, Y. Zhang, K. Schmidt-Rohr, M. Hong, pH-dependent conformation, dynamics, and aromatic interaction of the gating tryptophan residue of the influenza M2 proton channel from solid-state NMR, Biophys J. 104 (2013) 1698-1708. 
1 [54] R. Männikkö, P.J. Stansfeld, A.S. Ashcroft, A.T. Hattersley, M.S. Sansom, S. Ellard,

[55] Y. Wu, G.A. Voth, Computational studies of proton transport through the M2 channel, FEBS Lett. 552 (2003) 23-27.

[56] L. Garneau, H. Klein, M.F. Lavoie, E. Brochiero, L. Parent, R. Sauvé, Aromaticaromatic interactions between residues in KCa3.1 pore helix and S5 transmembrane segment control the channel gating process, J Gen Physiol. 143 (2014) 289-307.

[57] B. Holst, R. Nygaard, L. Valentin-Hansen, A. Bach, M.S. Engelstoft, P.S. Petersen, T.M. Frimurer, T.W. Schwartz, A conserved aromatic lock for the tryptophan rotameric switch in TM-VI of seven-transmembrane receptors, J Biol Chem. 285 (2010) 39733985.

[58] J.A. Ballesteros, X. Deupi, M. Olivella, E.E. Haaksma, L. Pardo, Serine and threonine residues bend alpha-helices in the chi(1) $=\mathrm{g}(-)$ conformation, Biophys J. 79 (2000) $2754-2760$.

[59] C. Del Val, S.H. White, A.N. Bondar, Ser/Thr motifs in transmembrane proteins: Conservation patterns and effects on local protein structure and dynamics. J Membr. Biol. 245 (2012) 717-730.

[60] E.K. O'Shea, J.D. Klemm, P.S. Kim, T. Alber, X-ray structure of the GCN4 leucine zipper, a two-stranded, parallel coiled coil, Science 254 (1991) 539-44.

[61] A.J. Gerc, A. Diepold, K. Trunk, M. Porter, C. Rickman, J.P. Armitage, N.R. StanleyWall, S.J. Coulthurst, Visualization of the Serratia Type VI secretion system reveals unprovoked attacks and dynamic assembly, Cell Rep. 12 (2015) 2131-2142.

[62] L. Roth, C. Nasarre, S. Dirrig-Grosch, D. Aunis, G. Crémel, P. Hubert, D. Bagnard, Transmembrane domain interactions control biological functions of neuropilin-1, Mol Biol Cell. 19 (2008) 646-654. 
1 [63] P. Hubert, P. Sawma, J.P. Duneau, J. Khao, J. Hénin, D. Bagnard, J. Sturgis, Singlespanning transmembrane domains in cell growth and cell-cell interactions: More than meets the eye?, Cell Adh Migr. 4 (2010) 313-324.

[64] A. Joshi, B. Kostiuk, A. Rogers, J. Teschler, S. Pukatzki, F.H. Yildiz, Rules of engagement: the Type VI secretion system in Vibrio cholerae, Trends Microbiol. 25 (2017) 267-279.

[65] T.G. Sana, B. Berni, S. Bleves, The T6SSs of Pseudomonas aeruginosa strain PAO1 and their effectors: beyond bacterial-cell targeting, Front Cell Infect Microbiol. 6 (2016) 61.

[66] J. Klockgether, B. Tümmler, Recent advances in understanding Pseudomonas aeruginosa as a pathogen, F1000Res. 6 (2017) 1261.

[67] T.G. Sana, N. Flaugnatti, K.A. Lugo, L.H. Lam, A. Jacobson, V. Baylot, E. Durand, L. Journet, E. Cascales, D.M. Monack, Salmonella Typhimurium utilizes a T6SS-mediated antibacterial weapon to establish in the host gut, Proc Natl Acad Sci U S A. 113 (2016) E5044-5051.

[68] W. Zhao, F. Caro, W. Robins, J.J. Mekalanos, Antagonism toward the intestinal microbiota and its effect on Vibrio cholerae virulence, Science. 359 (2018) 210-213.

[69] W.P. Russ, D.M. Engelman, The GxxxG motif: a framework for transmembrane helixhelix association, J Mol Biol. 296 (2000) 911-919.

[70] Y.R. Brunet, C.S. Bernard, M. Gavioli, R. Lloubes, E. Cascales, An epigenetic switch involving overlapping Fur and DNA methylation optimizes expression of a type VI secretion gene cluster, PLoS Genetics 7 (2011) e1002205.

[71] F. van den Ent, J. Lowe, RF cloning: a restriction-free method for inserting target genes into plasmids, J. Biochem. Biophys. Meth. 67 (2006) 67-74.

[72] A. Zaslaver, A. Bren, M. Ronen, S. Itzkovitz, I. Kikoin, S. Shavit, W. Liebermeister, M.G. Surette, U. Alon, A comprehensive library of fluorescent transcriptional reporters for Escherichia coli, Nat Methods. 3 (2006) 623-628. 
1 [73] E. Cascales, R. Lloubès, J.N. Sturgis, The TolQ-TolR proteins energize TolA and share 2 homologies with the flagellar motor proteins MotA-MotB, Mol Microbiol. 42 (2001) 795-807.

[74] J. Schindelin, I. Arganda-Carreras, E. Frise, V. Kaynig, M. Longair, T. Pietzsch, S. Preibisch, C. Rueden, S. Saalfeld, B. Schmid, J.Y. Tinevez, D.J. White, V. Hartenstein, K. Eliceiri, P. Tomancak, A. Cardona A, Fiji: an open-source platform for biologicalimage analysis, Nat Methods. 9 (2012) 676-682.

[75] R. Keller, Computer aided resonance assignment tutorial, (2014) http://cara.nmr.ch/

[76] N. Guex, M.C. Peitsch, SWISS-MODEL and the Swiss-PdbViewer: an environment for comparative protein modeling, Electrophoresis 18 (1997) 2714-2723

[77] S. Jo, J.B. Lim, J.B. Klauda, W. Im, CHARMM-GUI membrane builder for mixed bilayers and its application to yeast membranes, Biophys J. 97 (2009) 50-58.

\section{Legend to Figures}

\section{Figure 1. Schematic representation of the T6SS membrane complex and TssJLM}

proteins. (A) Representation of the T6SS highlighting the cytoplasmic tail structure anchored to the cell envelope by the membrane complex. (B) Negative-stain electron microscopy structural model of the TssJLM membrane complex [14]. (C) Localisation, topology and structure of the TssJ outer membrane lipoprotein, and of the TssL and TssM inner membrane proteins. IM, inner membrane; OM, outer membrane.

Figure 2. TssL ${ }^{\text {TM }}$ dimerizes. TOXCAT assay. NT316 reporter cells producing the indicated ToxR-X-MBP fusions (empty, no TMH sequence cloned; GpA ${ }^{\mathrm{TM}}$, Glycophorin A TMH; $\mathrm{GpA}^{\mathrm{TM}(\mathrm{G} 83 \mathrm{I})}, \mathrm{GpA}^{\mathrm{TM}}$ with G83I substitution; TssL ${ }^{\mathrm{TM}}$, TssL TMH) were streaked on M9 minimal medium supplemented with glucose (A) or maltose (B) as sole carbon source. 
1 Resistance to chloramphenicol (cam) conferred by the TM-mediated dimerization of the

2 ToxR transcriptional regulator was measured by the disk assay $(\mathrm{C}$, halo diameter mean \pm

3 standard deviation [SD] in $\mathrm{mm}$ ) and quantitative chloramphenicol acetyltransferase (Cat)

4 assay (D). The mean (value relative to the value obtained for the "empty" vector) is indicated 5 on top on each bar.

6 Figure 3. Thr194 and Trp199 contribute to TssL ${ }^{\text {TM }}$ dimerization. (A) Sequence of the

$7 \mathrm{TssL}^{\mathrm{TM}}$ peptide. The numbering is relative to the full-length TssL protein. The transmembrane 8 segment is indicated by the green frame and the leucine residues participating to a putative 9 leucine zipper are coloured red. The two leucine residues substituted in the LALA mutant 10 ware underlined. (B) TOXCAT disk assay of the $\mathrm{TssL}^{\mathrm{TM}}$ fragment and its LALA derivative.

11 The diameter of the halo is indicated on bottom (mean $\pm \mathrm{SD}$ in $\mathrm{mm}$ ). The quantitative Cat assay measures are reported in panel D. TOXCAT disk (C) and quantitative Cat (D) assays of the $\mathrm{TssL}^{\mathrm{TM}}$ fragments bearing the indicated substitutions. The level of activity corresponding to the empty plasmid is indicated by the red dotted line.

Figure 4. TM-mediated contacts stabilize TssL dimer on SDS-PAGE. $2 \times 10^{8} \Delta t s s L$ cells producing FLAG-tagged full-length TssL or the indicated TssL variants (TssL $L_{C}$, cytoplasmic domain of TssL; T194W and W199A, substitution variants of the TM Thr194 and Trp199 residues respectively) were subjected to $12.5 \%$ SDS-PAGE and immunoblotting with the monoclonal anti-FLAG antibody. The position of TssL, TssL $\mathrm{C}_{\mathrm{C}}$ and TssL dimers (*) are indicated on right. Molecular weight markers are indicated on left.

Figure 5. The Trp185 and Trp199 rings are protected in NMR Hydrogen-Deuterium exchange experiments. (A) Zoom-in of the $\mathrm{H}_{\mathrm{N}} / \mathrm{H}_{\mathrm{N}}$ region of the NOESY spectrum of the TssL $^{\mathrm{TM}}$ peptide $(2 \mathrm{mM})$ at $300 \mathrm{~K}$. The NMR sample was in phosphate buffer $\mathrm{pH} 4.5$ and SDS 
$11 \%\left(\mathrm{H}_{2} \mathrm{O}\right.$ conditions). Cross peaks (nuclear Overhauser effects) corresponding to coupling

2 between $\mathrm{H}_{\mathrm{N}}$ of residues $\mathrm{i}$ and $\mathrm{i}+1$ are observed. The assignment is shown in Table $\mathrm{S} 1$ and Fig.

3 S1. (B and C) Overlay of the NOESY spectra of the TssL ${ }^{\mathrm{TM}}$ peptide in $\mathrm{H}_{2} \mathrm{O}$ (blue) and $\mathrm{D}_{2} \mathrm{O}$

4 (red). $\mathrm{H}_{\mathrm{N}}$ chemical shift assignment was indicated by residues sequence numbering. In $\mathrm{D}_{2} \mathrm{O}$,

5 the exposed protons are exchanged with Deuterium and thus disappeared; however the

6 protected protons are not exchanged and thus are still observable. In (B) the regions of $\mathrm{H}_{\mathrm{N}}(\mathrm{x}$

7 axis) $/ \mathrm{H}_{\mathrm{CA}}$ (y axis) correlations is shown, and in (C) $\mathrm{H}_{\mathrm{N}}$ of rings of tryptophan residues are

8 shown. (D) Sequence of the TssL ${ }^{\mathrm{TM}}$ peptide. The numbering is relative to the full-length

9 TssL protein. The transmembrane segment is indicated by the green frame, and the tryptophan

10 residues protected in H-D exchange experiments (Trp185 and Trp199) are coloured red. (E)

11 Structural model of the $\mathrm{TssL}^{\mathrm{TM}}$ monomer in SDS micelles. The orientation of the

12 transmembrane segment relative to the membrane is shown. Residues located at the $\operatorname{TssL}^{\mathrm{TM}}$

13 interface (indicated on right), as well as residue Thr194 are represented by spheres

14 (tryptophan residues in grey, leucine zipper residues in pink). (F) Representation of the 15 structural model of Tss $\mathrm{L}^{\mathrm{TM}}$ dimer in SDS micelles obtained by MD simulations of the TssL ${ }^{\mathrm{TM}}$

16 monomer. The orientation of the two transmembrane segments relative to the membrane is

17 shown. The Trp185, Trp188 and Trp199 rings are represented by spheres and pointed with 18 arrowheads, highlighting the packing of the two Trp199 rings at the periplasmic side and of 19 the four rings from Trp185 and Trp188 at the cytoplasmic side.

20 Figure 6. The Trp185 and Trp199 rings control tight packing of the $\operatorname{TssL}^{\mathrm{TM}}$ dimer. (A) 21 Superimposition of the averaged structures of $\mathrm{TssL}^{\mathrm{TM}}$ monomerA obtained from the 22 molecular dynamic simulations of 8 different TssL ${ }^{\mathrm{TM}}$ dimers. The Thr194 and Trp199 23 residues are shown in spheres. (B and C) For the eight simulations of the dimer, (labeled from $240^{\circ}$ to $350^{\circ}$ ), the superimposed structure of monomer A (ribbon) is represented at the center. 25 The Thr194 and Trp199 side chains corresponding to the reference simulation $\left(0^{\circ}\right)$ are shown 
1 in spheres. The trajectory traces of the Thr194 (B) and Trp199 (C) side chains of monomer B

2 are shown at the periphery of the central helix. (D and E) Top panels: distances (in nm)

3 between the two residues Y184 (black), Trp185 (red), Trp188 (green), Thr194 (blue), or

4 Trp199 (yellow) in the dimer during 100-ns MD simulations starting from the $0^{\circ}$ (D) and $350^{\circ}$

5 (E) conformations. Bottom panels: final structures obtained after the 100-ns MD simulations

6 starting from the $0^{\circ}$ (D) and $350^{\circ}$ (E) conformations. Residues are shown in spheres with the

7 same color code than top panels.

8 Figure 7. Trp199 is required for T6SS function. (A) Hcp release assay. The cell pellet (C)

9 and supernatant (S) fractions from $5 \times 10^{8}$ cells of the wild-type 17-2 or $\Delta t s s L$ derivative, or $10 \Delta t s s L$ producing FLAG-tagged full-length TssL $(t s s L+)$ or the indicated TssL variants, and 11 HA-tagged Hcp, were subjected to $12.5 \%$ SDS-PAGE and immunoblotted with anti-TolB 12 (periplasmic leakage control, upper panel), anti-HA (to detect Hcp, middle panel) and anti13 FLAG (to detect TssL, lower panel) antibodies. Molecular weigh markers are indicated on the left. (B) Anti-bacterial activity. The indicated attacker strains were mixed with kanamycin resistant $\mathrm{GFP}^{+}$prey cells and spotted on SIM medium. After incubation, the fluorescent image 16 of the spot was recorded (middle panel) and level of surviving prey cells was estimated by 17 measuring the fluorescence (upper graph) and by counting the number of colonies on kanamycin plates (lower graph).

Figure 8. Trp199 stabilizes TssL. (A) $5 \times 10^{8}$-cell samples of the $\Delta t s s L$ strain producing

20 FLAG-tagged full-length TssL or the indicated TssL variants were harvested at the indicated time (in min) after protein synthesis arrest and were subjected to $12.5 \%$ SDS-PAGE and immunoblotting with anti-FLAG antibody, and secondary antibody coupled to AlexaFluor 680. Molecular weigh markers are indicated on the left. The relative intensity of each band 
1 was plotted against time (B) and half-times of TssL and TssL variants (in min) were

2 calculated from the slope of the first-order decay function (C).

3 Figure 9. Trp199-mediated $\operatorname{TssL}^{\mathrm{TM}}$ dimerization is required for TssL stability and T6SS

4 function. (A) Schematic representation of the TssL-W199A-F $F_{V}$ chimera protein. The TssL 5 full-length protein carrying the W199A substitution (schematized with the red star) was fused 6 to the FKBP F36V variant domain $\left(\mathrm{F}_{\mathrm{V}}\right.$, green). $\mathrm{F}_{\mathrm{V}}$ dimerization could be induced by the 7 rapamycin homologue AP20187 (AP, pink diamond). P, periplasm; C, cytoplasm. (B) 8 TOXCAT disk (upper panel) and quantitative Cat (lower panel) assays of W199A TssL ${ }^{\mathrm{TM}}$ 9 fused to $\mathrm{F}_{\mathrm{V}}\left(\mathrm{W} 199 \mathrm{~A}-\mathrm{F}_{\mathrm{V}}\right)$ in absence $(-)$ or presence $(+)$ of the FKBP ligand inducer AP20187 10 (AP). (C) Hcp release assay. The cell pellet (C) and supernatant (S) fractions from $5 \times 10^{8}$ $11 \Delta t s s L$ cells producing FLAG-tagged W199A-F $\mathrm{F}_{\mathrm{V}}$ and HA-tagged Hcp, grown in absence (-) or 12 presence $(+)$ of AP were subjected to $12.5 \%$ SDS-PAGE and immunoblotted with anti-TolB 13 (periplasmic leakage control, upper panel), anti-HA (to detect Hcp, middle panel) and antiFLAG (to detect TssL-F , lower panel) antibodies. Molecular weigh markers are indicated on the left. (D) Anti-bacterial activity. The $\Delta t s s L$ attacker strain producing W199A-F $\mathrm{F}_{\mathrm{V}}$ was mixed with kanamycin resistant $\mathrm{GFP}^{+}$prey cells and spotted on SIM medium supplemented

$17(+)$ or not (-) with AP. After incubation, the fluorescent image of the spot was recorded 18 (middle panel) and level of surviving prey cells was estimated by measuring the fluorescence (upper graph) and by counting the number of colonies on kanamycin plates (lower graph). (E) $5 \times 10^{8}$-cell samples of the $\Delta t s s L$ strain producing TssL-W199A- $\mathrm{F}_{\mathrm{V}}$, grown in absence (-) or presence $(+)$ of AP were harvested at the indicated time (in min) after protein synthesis arrest and were subjected to $12.5 \%$ SDS-PAGE and immunoblotting with anti-FLAG antibody, and secondary antibody coupled to AlexaFluor ${ }^{\circledR}$ 680. Molecular weigh markers are indicated on

24 the left. The relative intensity of each TssL-F $F_{V}$ band was plotted against time (F) and halftimes of W199A-F $F_{V}$ were calculated from the slope of the first-order decay function $(\mathrm{G})$. 
1 Figure 10. Sequence alignment of the TMHs from selected TssL homologues. The

2 sequences of the TMHs of the T6SS-associated TssL proteins and T4bSS-associated $3 \mathrm{IcmH} /$ DotU proteins from strains listed on left (phylogeny realised based on the full-length

4 TssL/IcmH sequences using the server at www.phylogeny.fr) were aligned using Clustal W

5 (right panel). The position of the TMH (from experimental data for EAEC [25] or identified 6 using the TMHMM and TMPred TMH prediction servers) is underlined in green. Tryptophan

7 and other aromatic residues located at the $\mathrm{N}$ - and $\mathrm{C}$-termini of the TMH sequences are

8 indicated in red and blue respectively. The residues participating in $\pi$ - $\pi$ packing for EAEC

9 TssL TMH dimerization (Y184, W185, W188 and W199; this study) are underlined. 


\section{Legend to Supplementary Figures}

2 Supplementary Figure S1. Assignment of the TssL ${ }^{\text {TM }}$ NOESY spectrum. The $H_{N} / H_{N}$

3 region of the NOESY spectrum of the TssL ${ }^{\mathrm{TM}}$ peptide in $\mathrm{H}_{2} \mathrm{O}$ is shown (same as Fig. 5A).

4 The cross peak correlations are indicated by sequential numbering of the TssL ${ }^{\mathrm{TM}}$ residues.

5 Supplementary Figure S2. Instability in the TssL ${ }^{\text {TM }}$ T194W mutant. (A) Contact map for 6 the TssL ${ }^{\mathrm{TM}}$ dimer (left) and the T194W mutant dimer (right). (B and C) Lateral (B, left panel)

7 and top (B, right panel) views and root mean square deviation (RMSD, in $\AA$ ) (C) of the

8 orientation of monomer B relative to the fixed monomer A during the course of the 20 -ns

9 molecular dynamics simulation of the T194W mutant. 


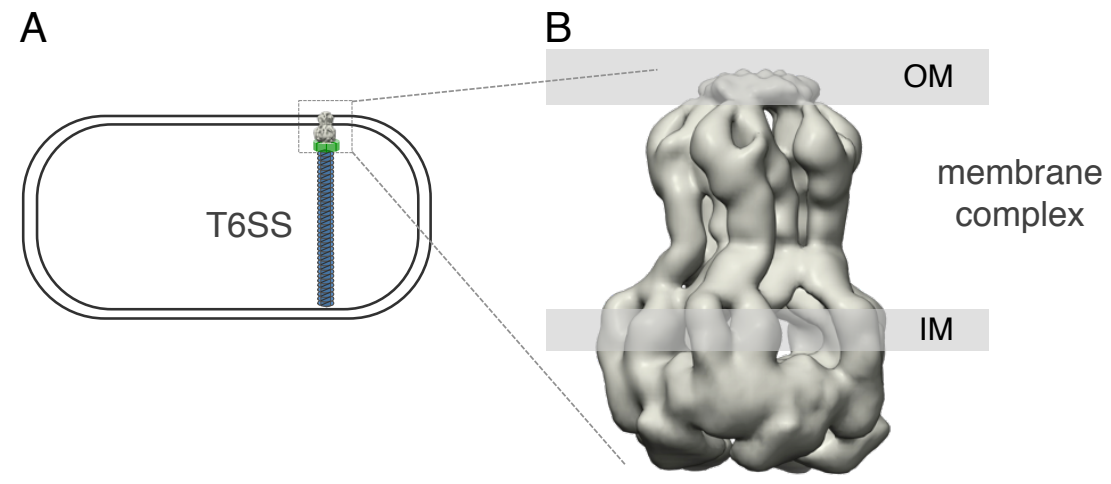

C

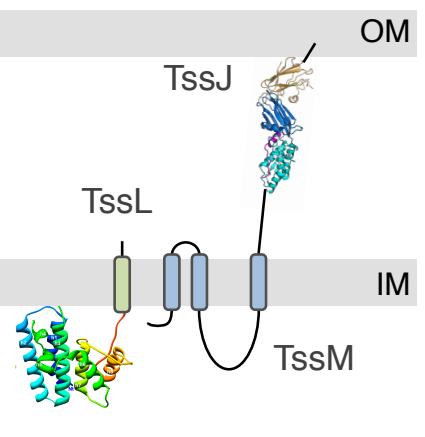

Figure 1 


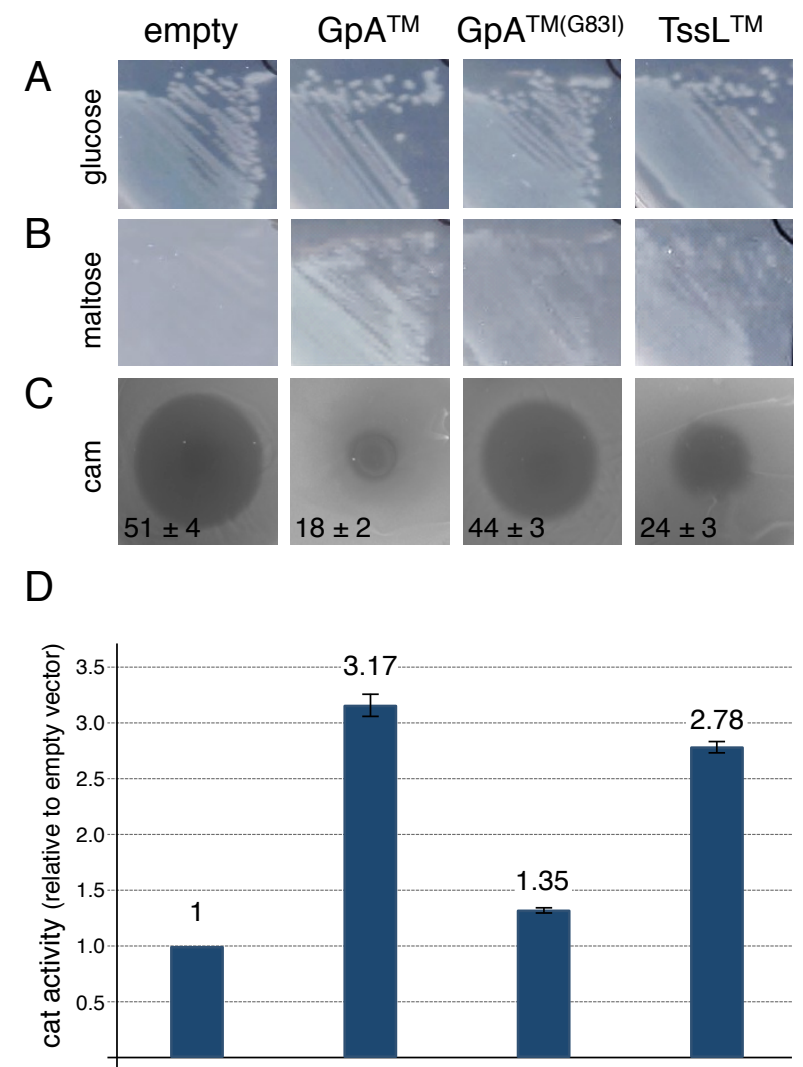

Figure 2 
A

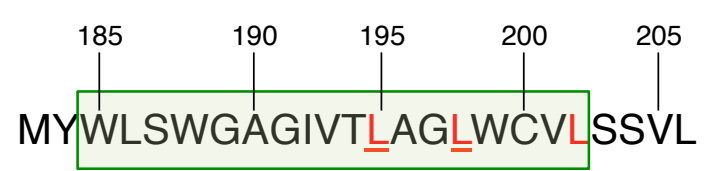

c
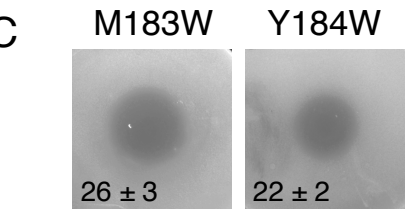

W185A

L186W
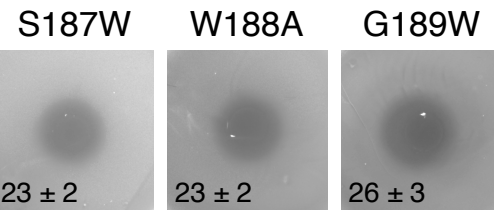

A190W
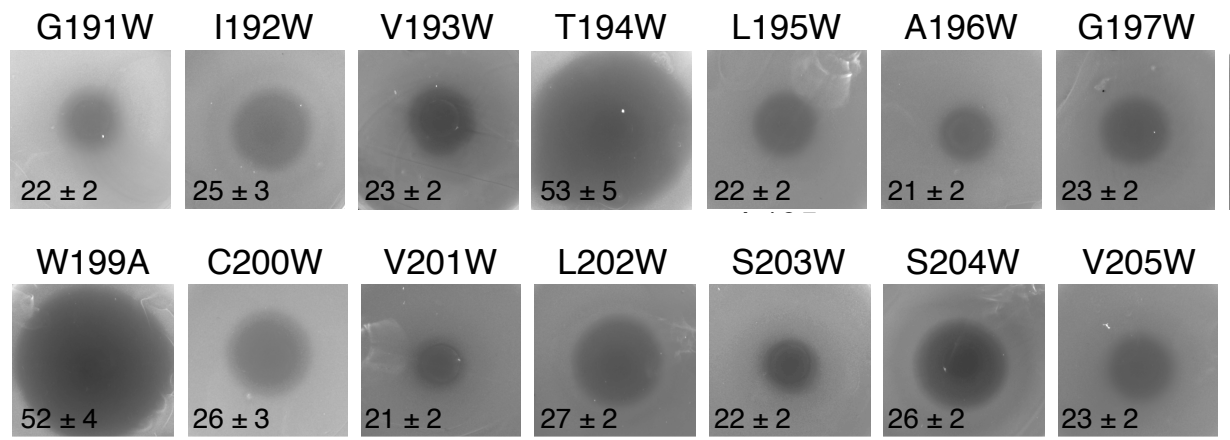

L198W
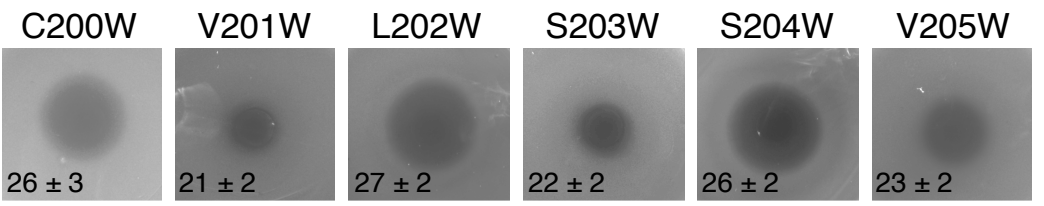

$22 \pm 2$

L206W

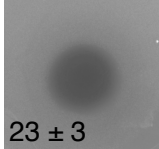

D

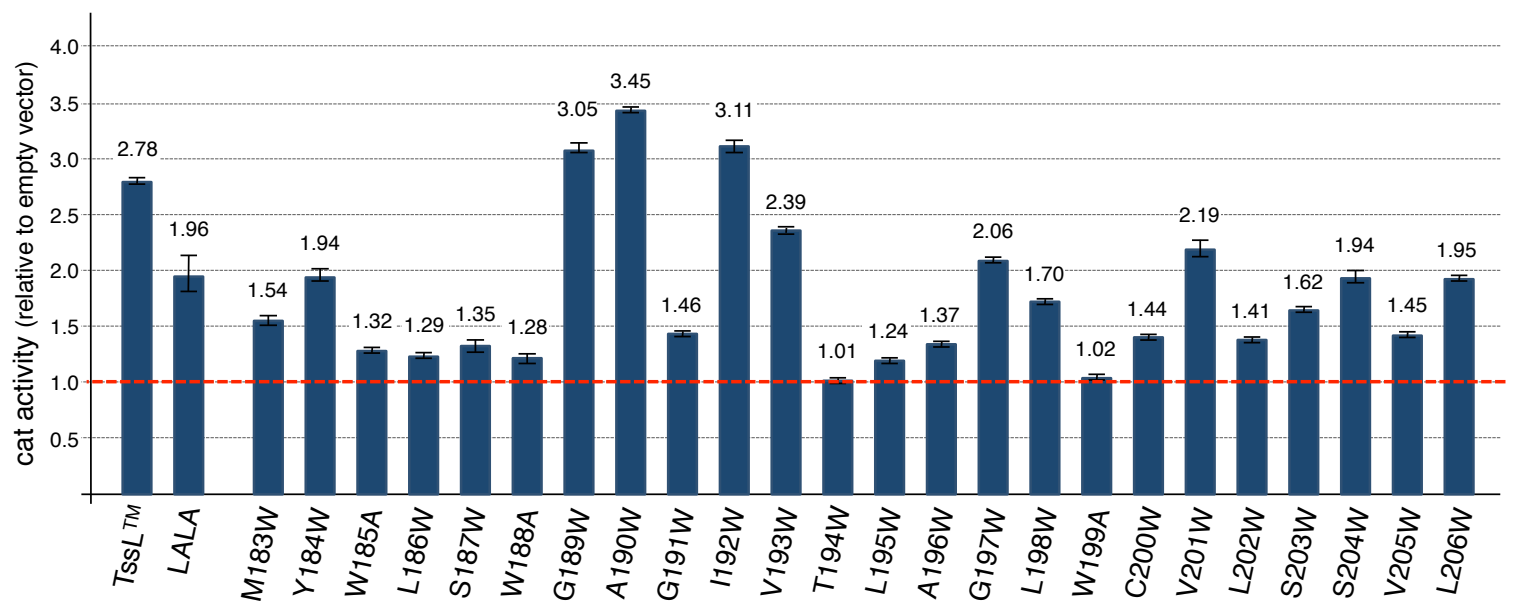




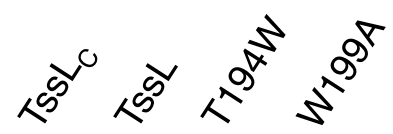

$64 \bullet$

50 •

$30 \bullet$

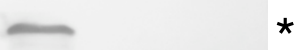

$\longrightarrow-T s s L$

$16 \bullet$

$$
\text { - TssL }
$$

Figure 4 

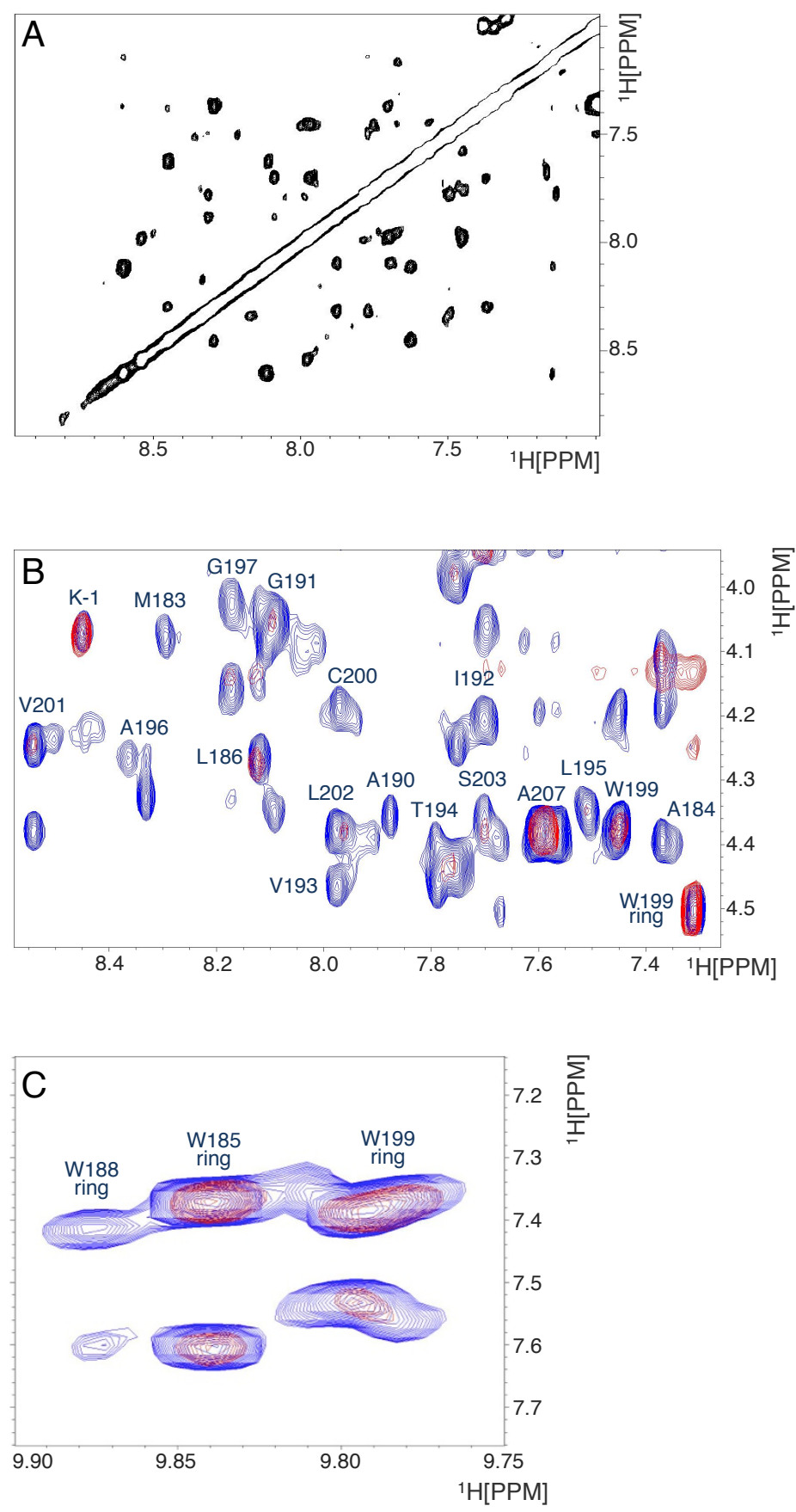

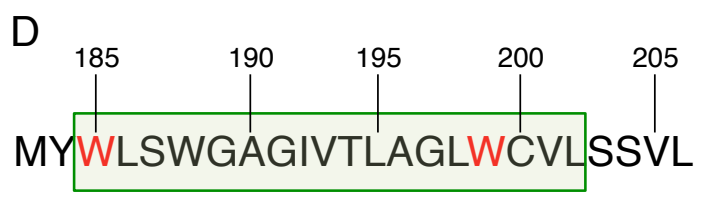

E TssL ${ }^{\mathrm{TM}}$ monomer

periplasm

cytoplasm

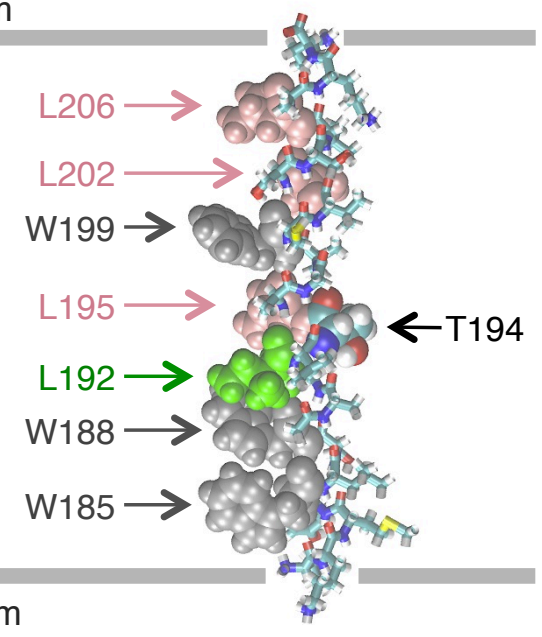

F

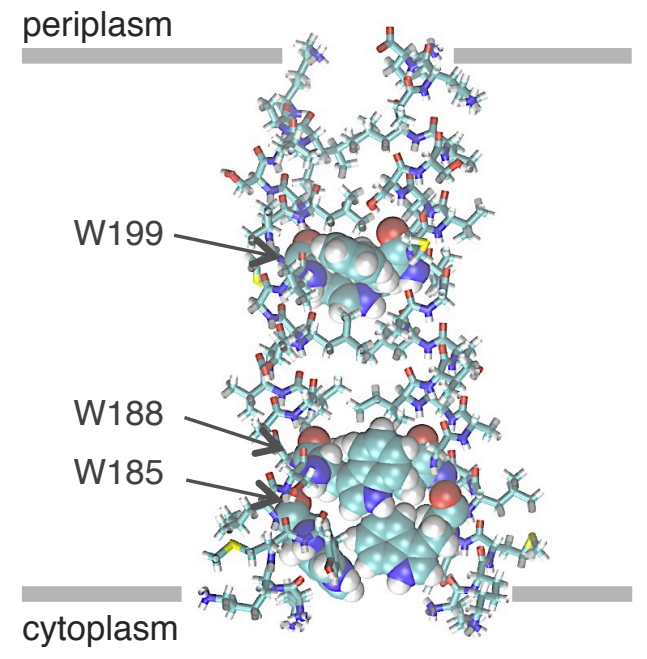


A

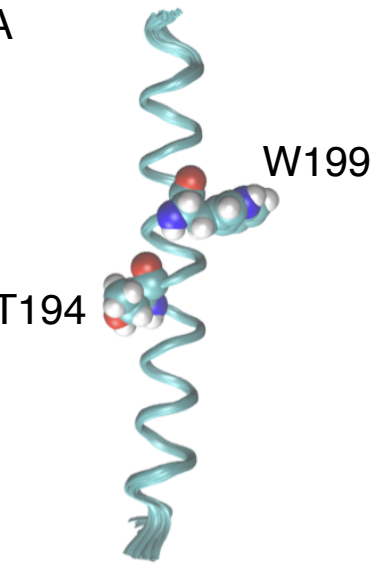

B

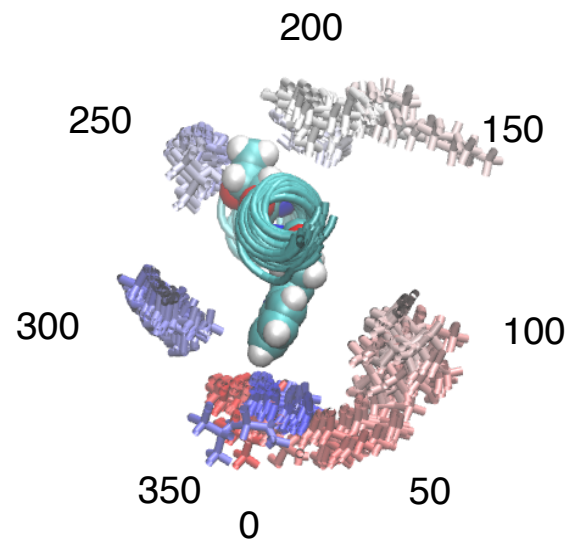

C

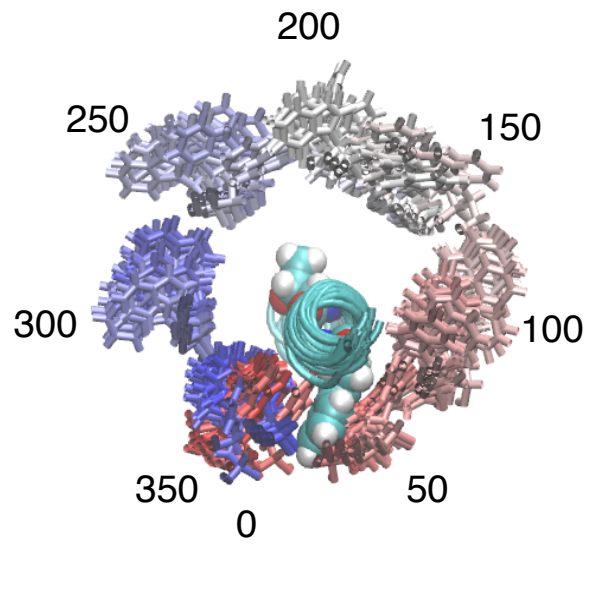

D
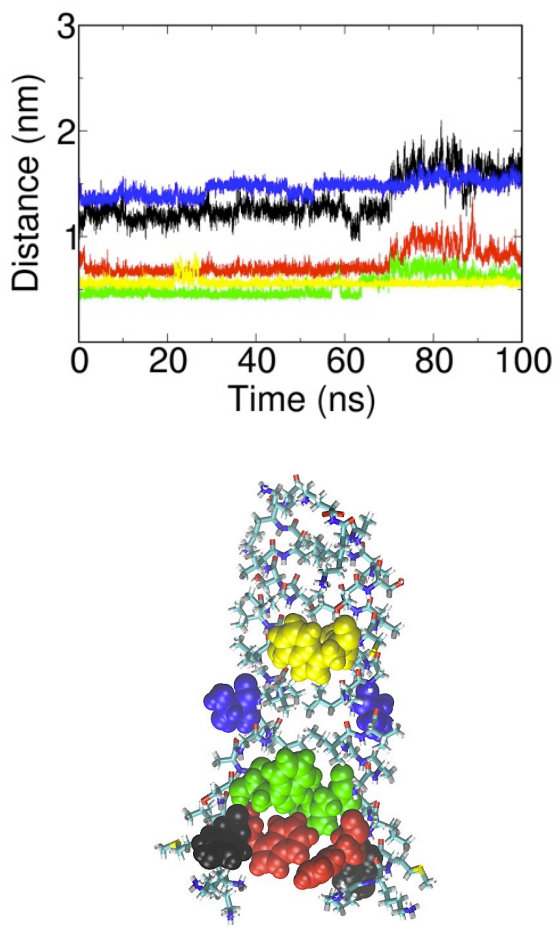

E

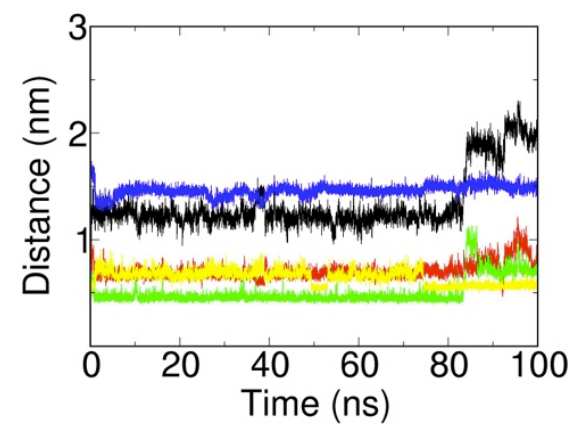

W199

T194

W188

W185

Y184

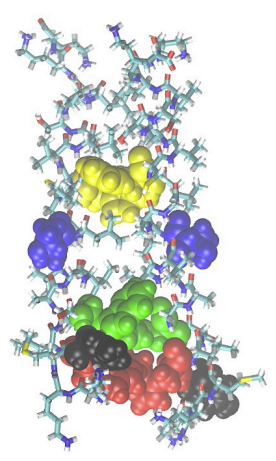



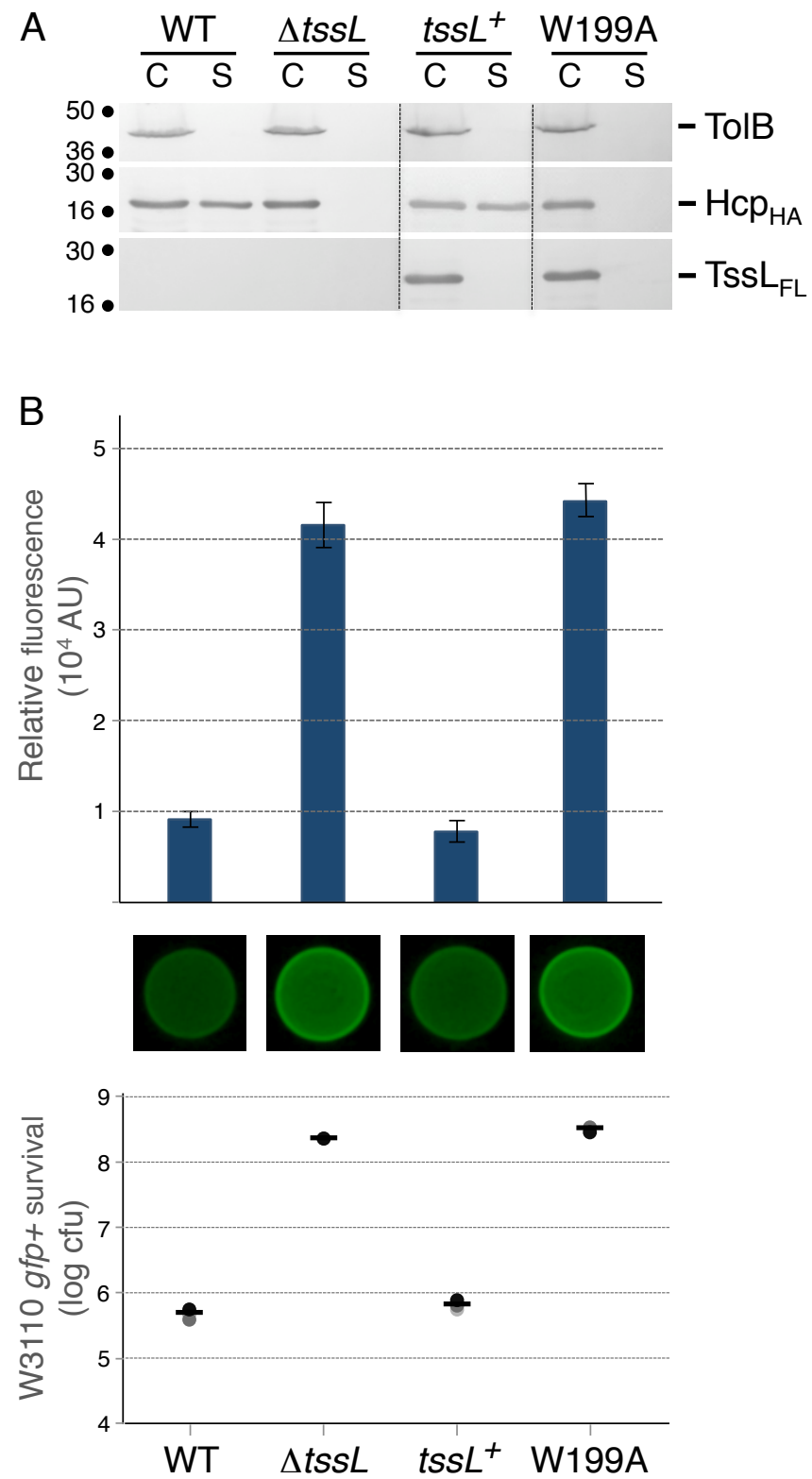
A

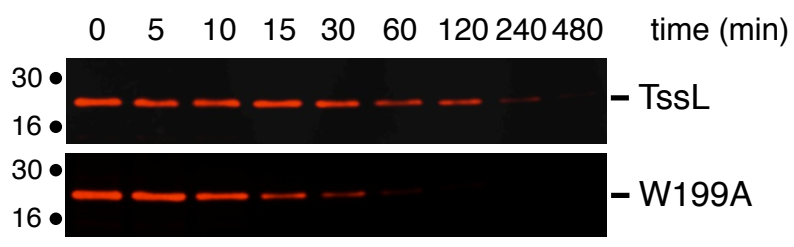

B

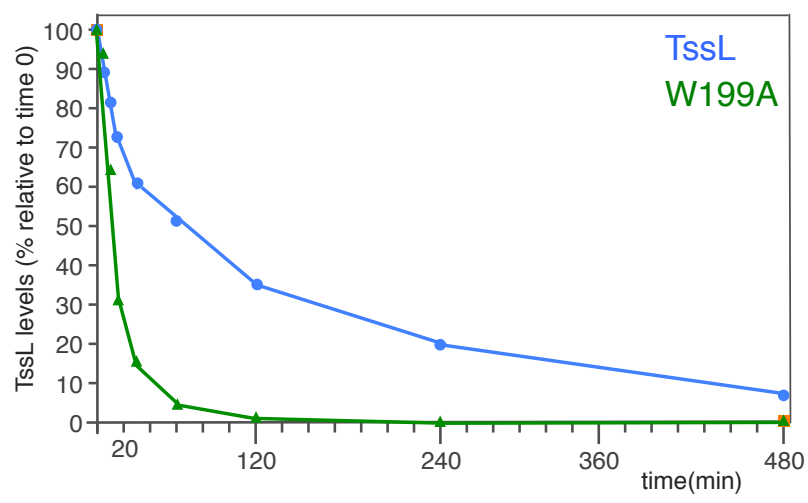

C

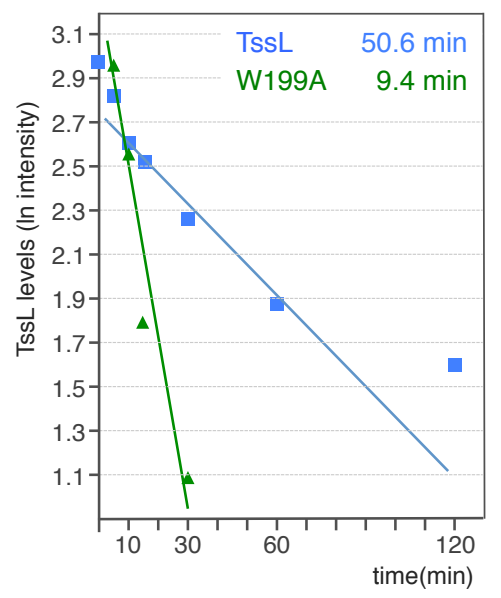

Figure 8 
A

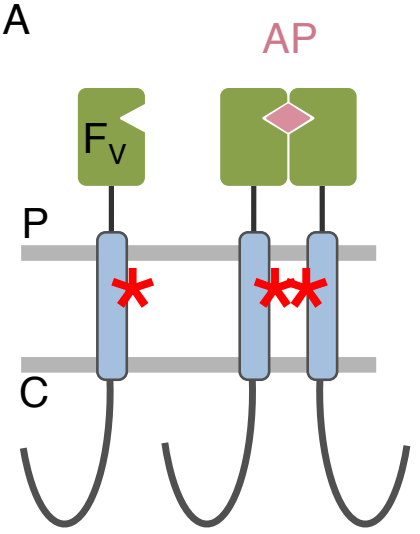

B
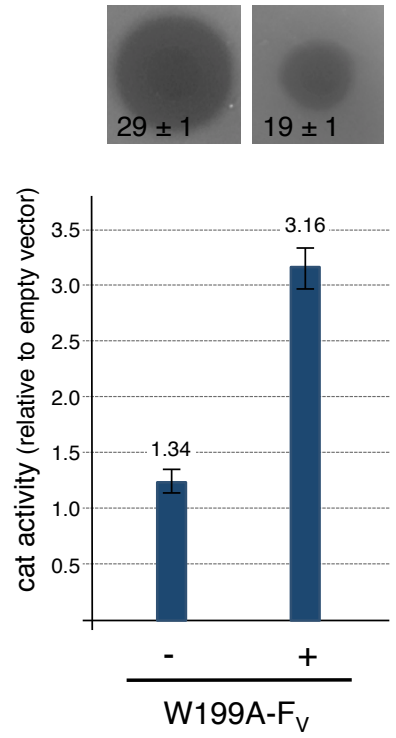

C W199A-F $\frac{-}{C \quad S} \frac{+}{C \quad S} A P$

50

$36 \bullet$

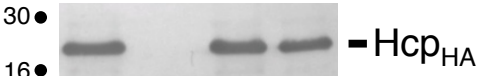

$16 \bullet$

50

$36 \bullet$

$30 \bullet$

D
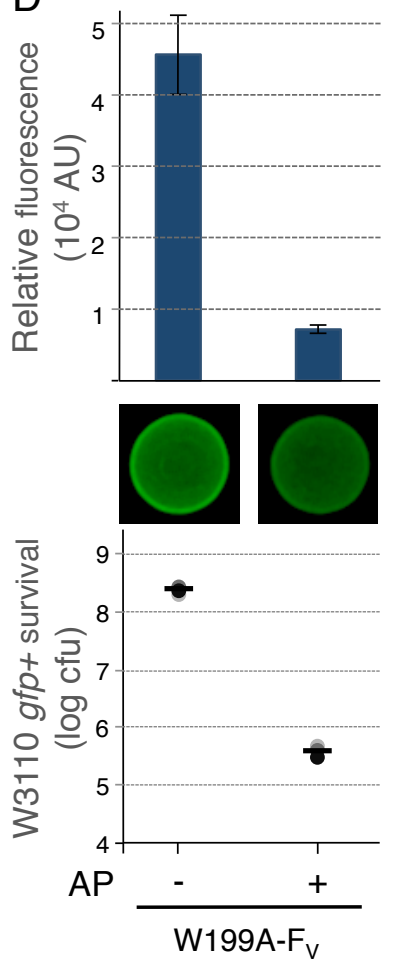

E

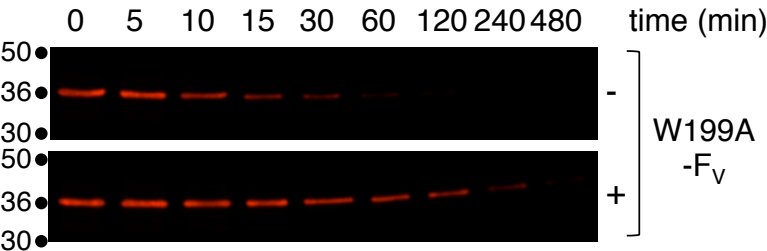

$\mathrm{F}$

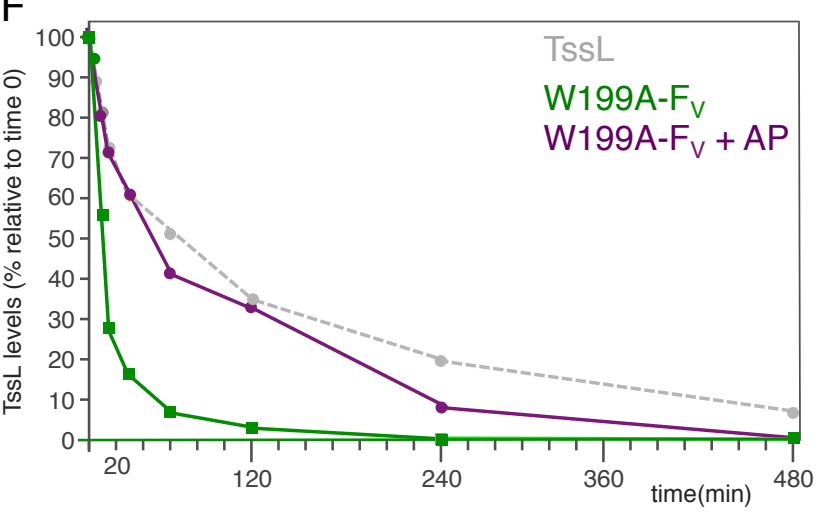

G

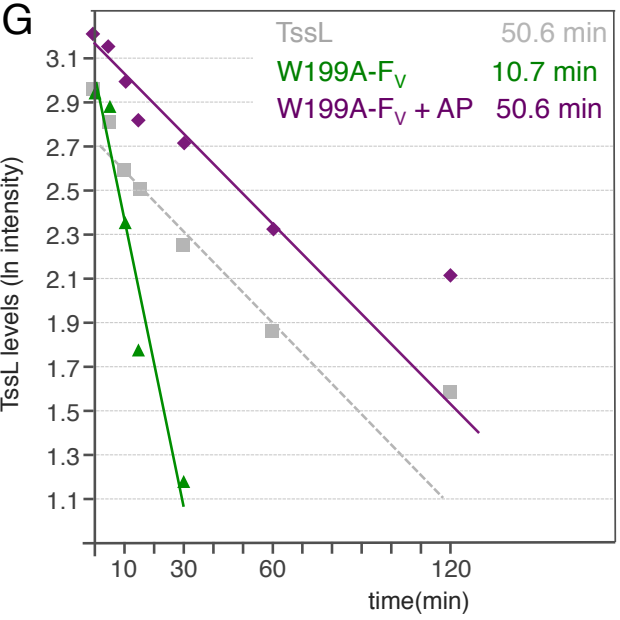




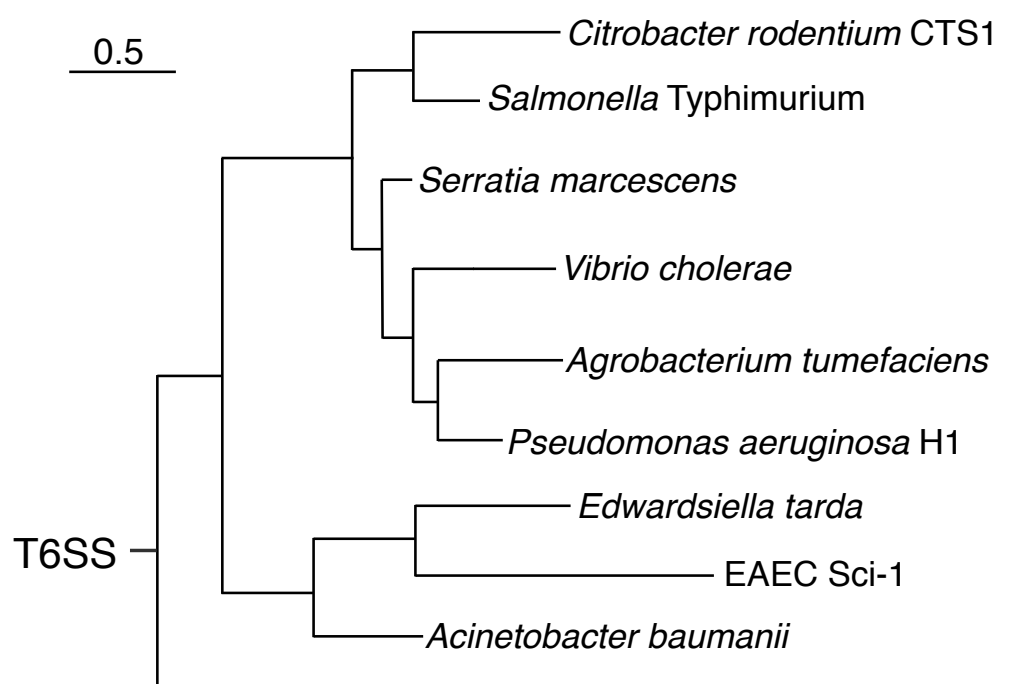

Francisella tularensis

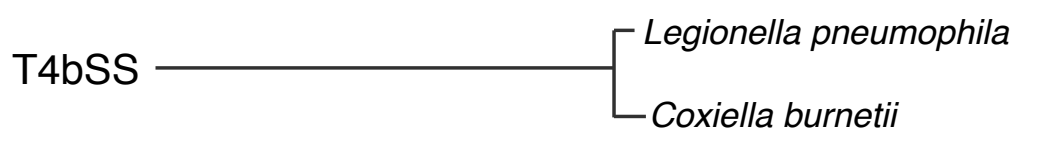

I PVRLSALCAGVLVVSSFLWCKFWLLS R-VPVWLSAGIALLAMLTLFGLYSHRM PLVPLWACVALTGFLASLLFIALNWRL

RQMPVWSVFAGFIVLWVGLFLGYSYVL TMAALWTLLSLMALAFALGYLFFTLSL QYLPPWVGIAIGLALLLALLFGLRMK FTTIVVPLVILVALYGILNWVLYEYAD RTM-YWLSWGGAGIVTLAGLWWCVLSSVL ELPFFWILIFLLIFALLTFAGLRFML TL-KFLLRIGVPLGLFLLSLLILLSW

YK-ATWITTIAAVGIVILAFVTSHVL RLPSVWATLGVTALVLIGIYLPYHFRL 


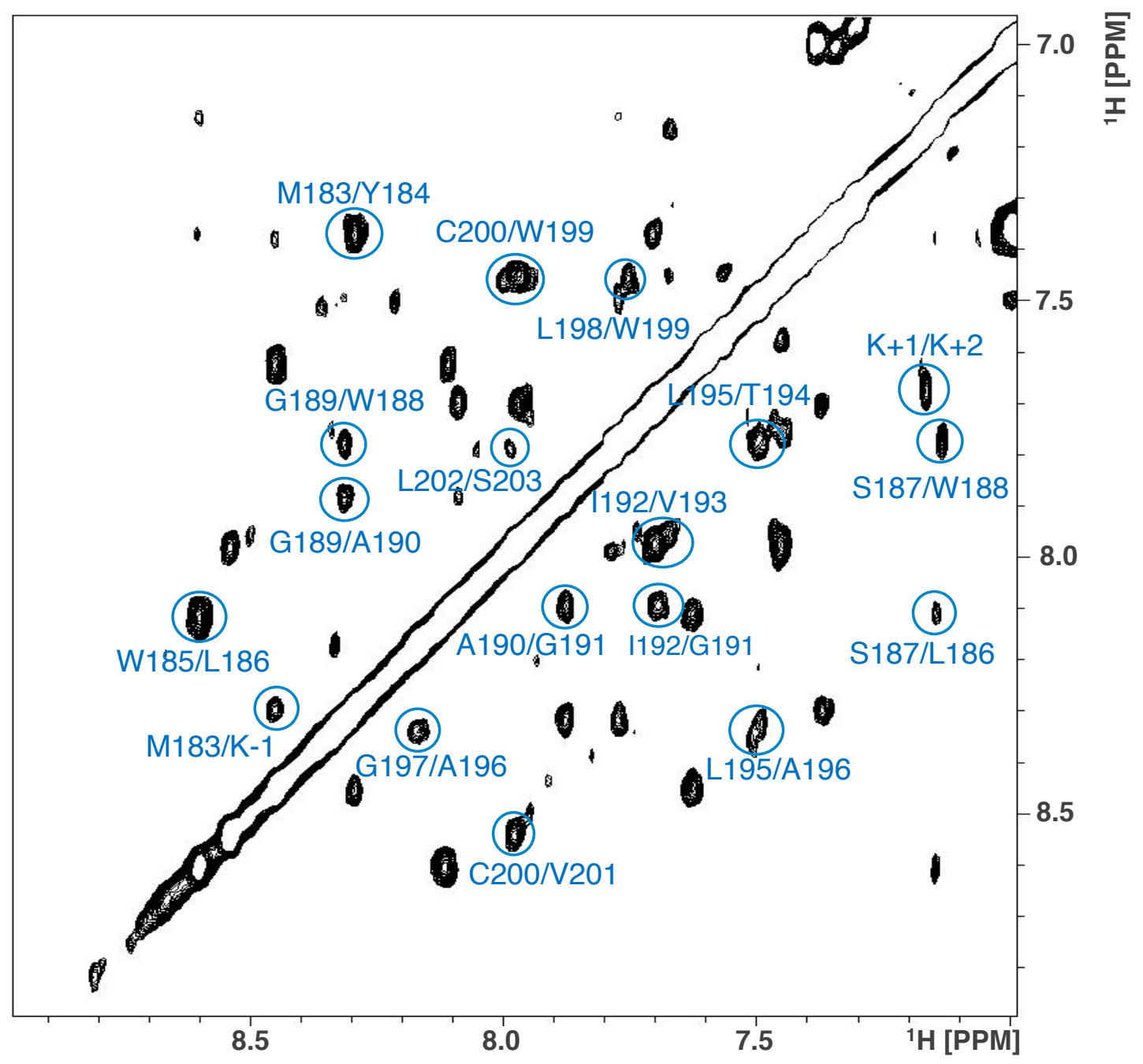

Figure S1 
A
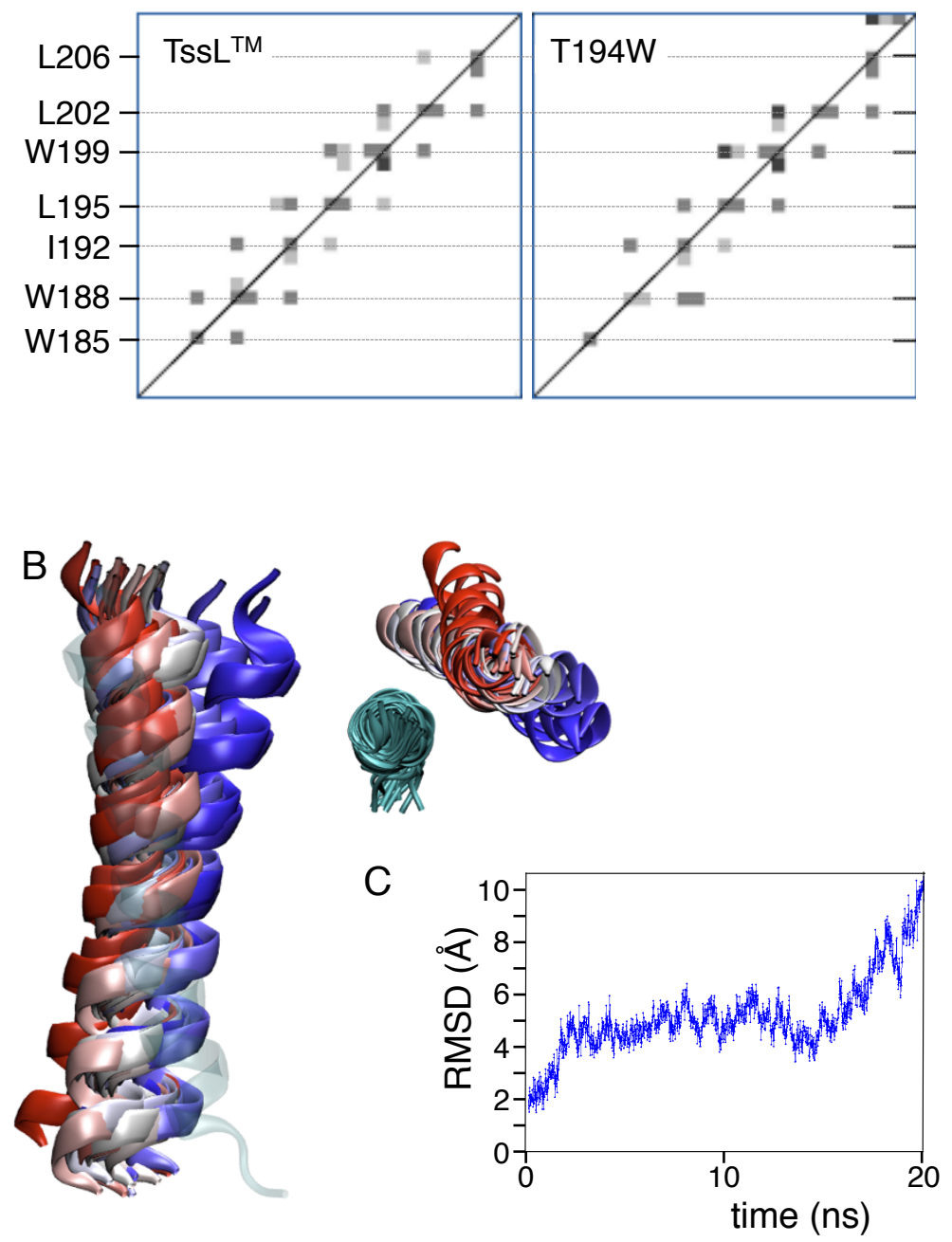

Figure S2 
Table S1. Sequential ${ }^{1} \mathrm{H}$ assignment of the $\mathrm{TssL}^{\mathrm{TM}}$ peptide (residues 183-207) at $300 \mathrm{~K}$ and $\mathrm{pH} 4.5$

\begin{tabular}{|c|c|c|c|c|}
\hline Residue & $\mathbf{H}_{\mathbf{N}}$ & Ha & Hb & Others \\
\hline Lys-2 & 7.62 & 3.70 & & Hg $1.03 ; 0.87 ;$ He 2.13 \\
\hline Lys-1 & 8.44 & 3.85 & & Hd 1.62; Hg 1.44;0.85 \\
\hline Met183 & 8.30 & 3.57 & $3.38 ; 1.60$ & \\
\hline Tyr184 & 7.36 & 4.50 & $3.96 ; 4.18$ & Hd 7.49; 7.00 Не 7.33; 6.90 \\
\hline Trp185 & 8.60 & 4.48 & $3.34 ; 3.3$ & Hd 7.14 Не 9.84; 7.81 Hh $6.98 \mathrm{~Hz} \mathrm{7.37;} 6.80$ \\
\hline Leu186 & 8.11 & 4.04 & $1.77 ; 1.67$ & Hg 1.52 Hd $0.90 ; 0.80$ \\
\hline Ser187 & 7.14 & 4.10 & 3.85 & \\
\hline Trp188 & 7.78 & 4.37 & $3.12 ; 3.29$ & Hd 7.18 Не 9.87; 7.48 Нh 6.99 Hz 7.37; 6.80 \\
\hline Gly189 & 8.32 & 3.64 & & \\
\hline Ala190 & 7.88 & 4.13 & 1.33 & \\
\hline Gly191 & 8.11 & 3.85 & & \\
\hline Ile192 & 7.71 & 3.99 & 1.75 & Hg $1.59 ; 1.34 ;$ Hd $0.64 ; 0.79$ \\
\hline Val193 & 7.97 & 4.23 & 2.2 & Hg 1.76 \\
\hline Thr194 & 7.78 & 4.16 & & Hg 1.42 \\
\hline Leu195 & 7.50 & 4.09 & 2.04 & Hd $0.89 ; \mathrm{Hg} 1.35$ \\
\hline Ala196 & 8.33 & 4.10 & 1.40 & \\
\hline Gly197 & 8.17 & 3.75 & 3.62 & \\
\hline Leu198 & 7.70 & 4.13 & & Hd 0.98;0.82; Hg 2.13 \\
\hline Trp199 & 7.45 & 4.18 & 4.02 & Hd 7.16; Нe 9.79;7.46; Hh 6.97; Hz 7.31;6.63 \\
\hline Cys200 & 7.99 & 3.98 & $2.18 ; 1.73$ & \\
\hline Val201 & 8.52 & 4.1 & 1.64 & Hg 1.31 \\
\hline Leu202 & 7.98 & 4.15 & $1.81 ; 1.60$ & Hg 1.31 \\
\hline Ser203 & 7.70 & 4.13 & 3.70 & \\
\hline Ser204 & 8.10 & 3.85 & $3.08 ; 3.00$ & \\
\hline Val205 & 8.21 & 3.75 & & Hg 1.6 \\
\hline Leu206 & 8.29 & 3.86 & $1.85 ; 1.61$ & Hg 1.38 \\
\hline Ala207 & 7.69 & 4.13 & 0.98 & \\
\hline Lys+1 & 7.67 & 4.26 & 1.69 & Hd $0.98 ;$ Не 2.12 \\
\hline Lys+2 & 7.16 & 4.67 & 1.60 & Не 2.89 \\
\hline
\end{tabular}




\section{Supplementary Table S2. Strains, plasmids and oligonucleotides used in this study.}

\section{Strains}

Strains

\section{E. coli $\mathrm{K}-12$}

DH5 $\alpha$

W3110

NT326

Enteroaggregative E. coli
$17-2$

$17-2 \Delta t s s L$
F-, $\Delta(\arg F-l a c) \mathrm{U} 169$, phoA, supE44, $\Delta(\mathrm{lacZ}) \mathrm{M} 15$, relA, endA, thi, hsdR

F-, lambda- IN(rrnD-rrnE) $1 r p h-1$

F-, $\Delta(\arg F-l a c) \mathrm{U} 169, r p s L, r e l A, r b s R, f l b B, p t s F, t h i, d e o C, \Delta m a l E 444, r e c A$
New England Biolabs

Laboratory collection
WT enteroaggregative Escherichia coli

17-2 deleted of the $t s s L$ gene of the $s c i 1$ T6SS gene cluster
Arlette Darfeuille-Michaud

[20]

\section{Plasmids}

\section{Vectors}

Description

Source

Expression vectors

pASK-IBA37

pASK-IBA37-TssL

pASK-IBA37-TssL $L_{C}$ cloning vector, Ptet, $\mathrm{Amp}^{\mathrm{R}}$

scil tssL cloned into pASK-IBA37, C-terminal FLAG epitope

Cytoplasmic domain (residues 1-183) of scil tss L cloned into pASK-IBA37,

N-terminal FLAG epitope
IBA Technology 
pASK-IBA37-TssL $L_{F L}-T 194 \mathrm{~W}$ pASK-IBA37-TssL $L_{F L}-W 199 A$ pASK-IBA37-TssL $L_{F L}-W 199 A-F K$ pOK-HcpHA pUA66-rrnb
Thr194 to Trp into pASK-IBA37-TssL Trp199 to Ala into pASK-IBA37-TssL

This study This study FK506 binding protein fused to TssL into pASK-IBA37-TssL This study scil hcp cloned into pOK12, C-terminal HA epitope

PrrnB ::gfpmut2 transcriptional fusion in pUA66, $\mathrm{Kan}^{\mathrm{R}}$

\section{Vectors for TOXCAT assay}

pcckan

pccGpA

pccGpG83I

pcc-TssL-TM

pcc-TssL-LALA

pcc-TssL-M183W

pcc-TssL-Y184W

pcc-TssL-W185A

pcc-TssL-L186W

pcc-TssL-S187W

pcc-TssL-W188A

pcc-TssL-G189W

pcc-TssL-A190W

pcc-TssL-G191W

pcc-TssL-I192W

pcc-TssL-V193W

pcc-TssL-T194W

pcc-TssL-L195W

pcc-TssL-A196W

pcc-TssL-G197W

pcc-TssL-L198W

pcc-TssL-W199A

pcc-TssL-C200W
ToxR-dependent expression of $c a t$

pcckan plasmid with glycophorin A TMH inserted between ToxR and MBP

[33]

Gly83 to Ile into pccGpA

pcckan plasmid with TssL TMH (residues 174-217) inserted between ToxR and MBP

[33]

Leu-195 and Leu-198 to Ala into pcc-TssL-TM

Met-183 to Trp into pcc-TssL-TM

Tyr-183 to Trp into pcc-TssL-TM

Trp-185 to Ala into pcc-TssL-TM

Leu-186 to Trp into pcc-TssL-TM

Ser-187 to Trp into pcc-TssL-TM

Trp-188 to Ala into pcc-TssL-TM

Gly-189 to Trp into pcc-TssL-TM

Ala-190 to Trp into pcc-TssL-TM

Gly-191 to Trp into pcc-TssL-TM

Ile-192 to Trp into pcc-TssL-TM

Val-193 to Trp into pcc-TssL-TM

Thr-194 to Trp into pcc-TssL-TM

Leu-195 to Trp into pcc-TssL-TM

Ala-196 to Trp into pcc-TssL-TM

Gly-197 to Trp into pcc-TssL-TM

Leu-198 to Trp into pcc-TssL-TM

Trp-199 to Ala into pcc-TssL-TM

Cys-200 to Trp into pcc-TssL-TM
This study

This study

This study

This study

This study

This study

This study

This study

This study

This study

This study

This study

This study

This study

This study

This study

This study

This study

This study

This study 


$\begin{array}{lll}\text { pcc-TssL-V201W } & \text { Val-201 to Trp into pcc-TssL-TM } & \text { This study } \\ \text { pcc-TssL-L202W } & \text { Leu-202 to Trp into pcc-TssL-TM } & \text { This study } \\ \text { pcc-TssL-S203W } & \text { Ser-203 to Trp into pcc-TssL-TM } & \text { This study } \\ \text { pcc-TssL-S204W } & \text { Ser-204 to Trp into pcc-TssL-TM } & \text { This study } \\ \text { pcc-TssL-V205W } & \text { Val-205 to Trp into pcc-TssL-TM } & \text { This study } \\ \text { pcc-TssL-L206W } & \text { Leu-206 to Trp into pcc-TssL-TM } & \text { This study } \\ \text { pcc-TssL-W199A-FK } & \text { FK506 binding protein fused to TssL-TM into pcc-TssL-W199 } & \text { This study }\end{array}$

\section{Oligonucleotides}

Name Destination $\quad$ Sequence $\left(5^{\prime} \rightarrow 3^{\prime}\right)$

\section{For plasmid construction}

\begin{tabular}{|c|c|c|}
\hline 5-TssLTM-NheI & insertion of TssL-TM into pcckan & GGTGGTGGTGCGTGCTAGCGCACCGGGTTACCGTG \\
\hline 3-TssLTM-BamHI & insertion of TssL-TM into pcckan & $\begin{array}{l}\text { CGTACGTGCATGGTTAGGATCCCTCCCTGCCCGGTA } \\
\text { AGCC }\end{array}$ \\
\hline 5-pcckn-L-TM-FKBP & FK506 binding protein fused to TssL-TM & $\begin{array}{c}\text { CAGGTGGCACGGCTTACCGGGCAGGGAGGGCTC } \\
\text { GAGATGGCTTCTAGAGGAGTGCAGGTG }\end{array}$ \\
\hline 3-pcckn-L-TM-FKBP & FK506 binding protein fused to TssL-TM & $\begin{array}{l}\text { GTTTAAAGCTGGATTGGCTTGGGTTGATCAGGA } \\
\text { TTCATTCCAGTTTTAGAACCTTCACATCG }\end{array}$ \\
\hline 5-IBA-TssL-LFKBP & FK506 binding protein fused to TssL & $\begin{array}{l}\text { CAGGTGGCACGGCTTACCGGGCAGGGACTCGAGATGG } \\
\text { CTTCTAGAGGAGTGCAGG }\end{array}$ \\
\hline 3-IBA-TssL-FKBP & FK506 binding protein fused to TssL & $\begin{array}{l}\text { GATGGTGATGCGATCCTCTGCTAGCTCATTCCAGTTTT } \\
\text { AGAACCTTCACATCGAAG }\end{array}$ \\
\hline
\end{tabular}

For site directed mutagenesis 
3-TssL-L195A-L198A

\begin{tabular}{ll} 
5-A-M183W & Met183 to Trp \\
5-A-Y184W & Tyr183 to Trp \\
5-A-W185A & Trp185 to Ala \\
5-A-L186W & Leu186 to Trp \\
\hline 5-A-S187W & Ser187 to Trp \\
3-A-M183-S187 & Trp188 to Ala \\
5-B-W188A & Gly189 to Trp \\
5-B-G189W & Ala190 to Trp \\
5-B-A190W & Gly191 to Trp \\
5-B-G191W & Ile192 to Trp \\
\hline 5-B-I192W & Val193 to Trp \\
\hline 3-B-W188-I192 & Thr194 to Trp \\
\hline 5-C-V193W & Leu195 to Trp \\
\hline 5-C-T194W & Ala196 to Trp \\
\hline 5-C-L195W & \\
\hline 5-C-A196W &
\end{tabular}

GAAGAAAGCACACACCAGGCACCAGCCGCCGTCACAA TACCCGCCCC

GTTACCGTGCTGGCAGAACGTGGTACTGGTTGTCATGG GGG

GTTACCGTGCTGGCAGAACGATGTGGTGGTTGTCAT GGGGGGCG

GTTACCGTGCTGGCAGAACGATGTACGCGTTGTCATG GGGGGCGGG

GTTACCGTGCTGGCAGAACGATGTACTGGTGGTCAT GGGGGGCGGGTAT

GTTACCGTGCTGGCAGAACGATGTACTGGTTGTGGTG GGGGGCGGGTATTGTG

TCTGCCAGCACGGTAACCCGGTGCACGCAC

GAACGATGTACTGGTTGTCAGCGGGGGCGGGTATTGT GAC

GAACGATGTACTGGTTGTCATGGTGGGCGGGTATTGTG ACG

GAACGATGTACTGGTTGTCATGGGGGTGGGGTATTGT GACGCT

GAACGATGTACTGGTTGTCATGGGGGGCGTGGATTGT GACGCTGGC

GAACGATGTACTGGTTGTCATGGGGGGCGGGTTGGGT GACGCTGGCTGG

CAACCAGTACATCGTTCTGCCAGCACGGTA

TGTCATGGGGGGCGGGTATTTGGACGCTGGCTGGTCTC TGG

TGTCATGGGGGGCGGGTATTGTGTGGCTGGCTGGTCTC TGGTG

TGTCATGGGGGGCGGGTATTGTGACGTGGGCTGGTCTC TGGTGTG

TGTCATGGGGGGCGGGTATTGTGACGCTGTGGGGTCTC TGGTGTGTG 


\begin{tabular}{|c|c|c|}
\hline 5-C-G197W & Gly197 to Trp & $\begin{array}{l}\text { TGTCATGGGGGGCGGGTATTGTGACGCTGGCTTGGCTC } \\
\text { TGGTGTGTGCTT }\end{array}$ \\
\hline 5-D-L198W & Leu198 to Trp & $\begin{array}{l}\text { GTATTGTGACGCTGGCTGGTTGGTGGTGTGTGCTTTCT } \\
\text { TCT }\end{array}$ \\
\hline 5-D-W199A & Trp199 to Ala & $\begin{array}{l}\text { GTATTGTGACGCTGGCTGGTCTCGCGTGTGTGCTTTCT } \\
\text { TCTGTG }\end{array}$ \\
\hline $5-\mathrm{D}-\mathrm{C} 200 \mathrm{~W}$ & Cys200 to Trp & $\begin{array}{l}\text { GTATTGTGACGCTGGCTGGTCTCTGGTGGGTGCTTTCT } \\
\text { TCTGTGCT }\end{array}$ \\
\hline 5-D-L202W & Leu202 to Trp & $\begin{array}{l}\text { GTATTGTGACGCTGGCTGGTCTCTGGTGTGTGTGGTCT } \\
\text { TCTGTGCTTGCAG }\end{array}$ \\
\hline 3-D-L198-L202 & & AGCCAGCGTCACAATACCCGCCCCCCATGA \\
\hline 5-E-S203W & Ser203 to Trp & $\begin{array}{l}\text { CTGGTCTCTGGTGTGTGCTTTGGTCTGTGCTTGCAGAC } \\
\text { CAG }\end{array}$ \\
\hline 5-E-S204W & Ser204 to Trp & $\begin{array}{l}\text { CTGGTCTCTGGTGTGTGCTTTCTTGGGTGCTTGCAGAC } \\
\text { CAGGTG }\end{array}$ \\
\hline 3-E-S203-L206 & & CACACACCAGAGACCAGCCAGCGTCACAAT \\
\hline
\end{tabular}

